\title{
Ionic Fluorogels for Remediation of Per- and Polyfluorinated Alkyl Substances from Water.
}

\author{
Elango Kumarasamy, ${ }^{1, \#}$ Irene M. Manning, ${ }^{1, \#}$ Leonard B. Collins, ${ }^{2}$ Orlando Coronell, ${ }^{*, 2}$ and Frank A. \\ Leibfarth*,1 \\ ${ }^{1}$ Department of Chemistry, University of North Carolina at Chapel Hill, Chapel Hill, NC, 27599, USA. \\ ${ }^{2}$ Department of Environmental Sciences and Engineering, Gillings School of Global Public Health, University of North Car- \\ olina at Chapel Hill, Chapel Hill, NC 27599, USA
}

\section{Supporting Information Placeholder}

PFAS are a class of fluorinated compounds that are widely used as surfactants in the production of poly(tetrafluoroethylene) (Teflon), as water/stain repellant coatings in consumer products, and as components of fire retardants in aqueous film forming foams. ${ }^{1-4}$ PFAS are distributed widely from contamination sites through waterways, ${ }^{5}$ and their long-term ecological persistence and adverse human health effects ${ }^{6-11}$ have resulted in increased regulatory attention to the concentration of PFAS in finished drinking water. ${ }^{12,13}$ The U.S.
Environmental Protection Agency has set a lifetime health advisory level of $70 \mathrm{ng} / \mathrm{L}$ for the combined concentration of perfluorooctanoic acid (PFOA) and perfluoro-1-octane sulfonic acid (PFOS) in drinking water. ${ }^{14,15}$ Consequently, PFOA and PFOS were phased out in the United States in 2015 and were replaced with short chain PFAS such as perfluoro-2-propoxypropanoic acid (GenX), ${ }^{16}$ which demonstrate similar bioaccumulation potential. ${ }^{12,17}$ Currently, the state of North Carolina has set an upper limit of $140 \mathrm{ng} / \mathrm{L}$ for GenX as an emerging contaminant in drinking water.

Current and emerging PFAS remediation technologies typically remove waterborne contaminants nonspecifically, resulting in saturation by non-fluorinated species. ${ }^{18,19}$ A primary challenge for developing a resin for PFAS remediation is that non-fluorinated organic and inorganic species are present in natural waters at three to eight orders of magnitude higher concentration than PFAS. ${ }^{20}$ For example, current PFAS remediation efforts using granular activated carbon (GAC) demonstrate substantial breakthrough at modest treatment volumes for short-chain PFAS. ${ }^{21}$ Furthermore, the binding affinities of organic contaminants to GAC is often higher than those of PFAS, which can result in PFAS leaching into filtered water over time. ${ }^{21,22}$ Emerging technologies include porous organic polymer adsorbents ${ }^{23-30}$ and ion exchange materials that contain a fluorinated component. ${ }^{31-34}$ While these materials show great promise for adsorbing long-chain PFAS, they are still at an early stage of development, have generally not been tested in real water, and display modest selectivity for short-chain PFAS. 
Scheme 1. Polymerization and Quaternization of Ionic Fluorogels.

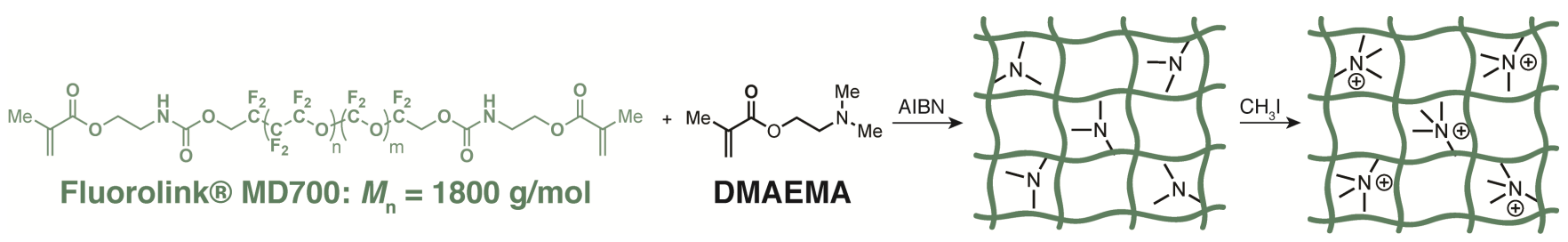

We identified a materials design platform to remediate PFAS from water by combining two complementary strategies - fluorophilic sorption and targeted ion exchange. Our conceptual approach leverages the fluorophilicity of PFAS to selectively partition these micropollutants into a resin, similar to the method commonly used to separate desired fluorous-tagged products or catalysts from complex reaction mixtures. ${ }^{35-37}$ In addition, we reasoned that the incorporation of a tunable density of charged functional groups would enable ion exchange and sequestration of charged PFAS. We chose perfluoropolyethers (PFPEs) as the fluorophilic matrix material for resin development. ${ }^{38}$ PFPEs are amorphous, low molecular weight perfluorinated oligomers that are synthesized from the gas phase without the use of perfluorinated surfactants. ${ }^{39,40}$ PFPEs can be synthesized using supercritical $\mathrm{CO}_{2}$ as a solvent ${ }^{41}$ and they are easier to oxidatively degrade than perfluorocarbons at the termination of their useful lifetime. ${ }^{42}$ The Ionic Fluorogels (IFs) described herein leverage a synergistic combination of fluorophilicity and ion exchange to generate high-performing and selective resins for PFAS remediation from natural water sources.

The synthesis of Ionic Fluorogels was achieved through thermally-initiated radical copolymerization initiated by azobisisobutyronitrile (AIBN) of a commercially available PFPE with methacrylate chain-end functionality (Fluorolink ${ }^{\circledR}$ MD 700) and an amine-containing monomer (2-dimethylaminoethyl methacrylate, DMAEMA) (Scheme 1). The composition of DMAEMA was varied from $10-60 \mathrm{wt} \%$ with respect to the total weight of the IF to generate a systematic library of materials that varies the ratio of fluorophilic and charged components. A portion of each formulation was subsequently treated with methyl iodide to access materials with quaternary ammonium groups that act as permanent charged species. Grinding and sieving the material provided a granular formulation with particle size between 75-125 microns for evaluation. This approach rapidly provides diverse IF formulations from the polymerization of commercially available components. A separate library of materials was prepared to act as negative controls in our structure-property studies. First, a PFPE elastomer with no electrostatic component (no DMAEMA) was polymerized. Second, non-fluorous ionic gels with charged groups but without a fluorous component were synthesized through the radical copolymerization of polyethylene glycol dimethacrylate (PEGMA $M_{\mathrm{n}}=750$ $\mathrm{g} / \mathrm{mol}$ ) and DMAEMA. This particular PEGMA was chosen to mimic a similar degree of polymerization between crosslinks as Fluorolink ${ }^{\circledR}$ MD 700.

The PFAS removal efficiency of each IF formulation was tested by conducting batch equilibrium adsorption experiments in simulated natural water, which was formulated by adding $200 \mathrm{mg} / \mathrm{L} \mathrm{NaCl}$ and $20 \mathrm{mg} / \mathrm{L}$ humic acid to deionized water. Three substrates that represent long chain (PFOA), short chain (perfluorohexanoic acid, PFHxA), and branched (GenX) PFAS were spiked into the matrix each at an environmentally relevant concentration $(1.0 \mu \mathrm{g} / \mathrm{L})$. After exposing the contaminated water sample to $10 \mathrm{mg} / \mathrm{L}$ of Ionic Fluorogel for 21 hours, PFAS removal efficiency was analyzed by liquid chromatography mass spectrometry (LC-MS). The results of this systematic study revealed valuable structureproperty information (illustrative results in Figure 1, complete results in Figure S2). Ionic Fluorogels containing tertiary amines (IF-X, X $=$ wt \% amine comonomer incorporation) demonstrated lower affinity for PFAS than the respective materials that contained quaternary ammonium groups $\left(\mathbf{I F}-\mathbf{X}+, \mathrm{X}^{+}=\mathrm{wt} \%\right.$ ammonium comonomer incorporation) across all formulations tested (Figure 1A), proving the importance of incorporating permanent charge. The highest performing formulations contained between 20 to $30 \mathrm{wt} \%$ ammonium comonomer incorporation (IF-20+ through IF-30+), demonstrating $>80 \%$ removal of short-chain PFAS, PFHxA and GenX. We hypothesize that these formulations have enough ammonium content to enable efficient wetting while still containing enough fluorous content to provide selective PFAS adsorption. 


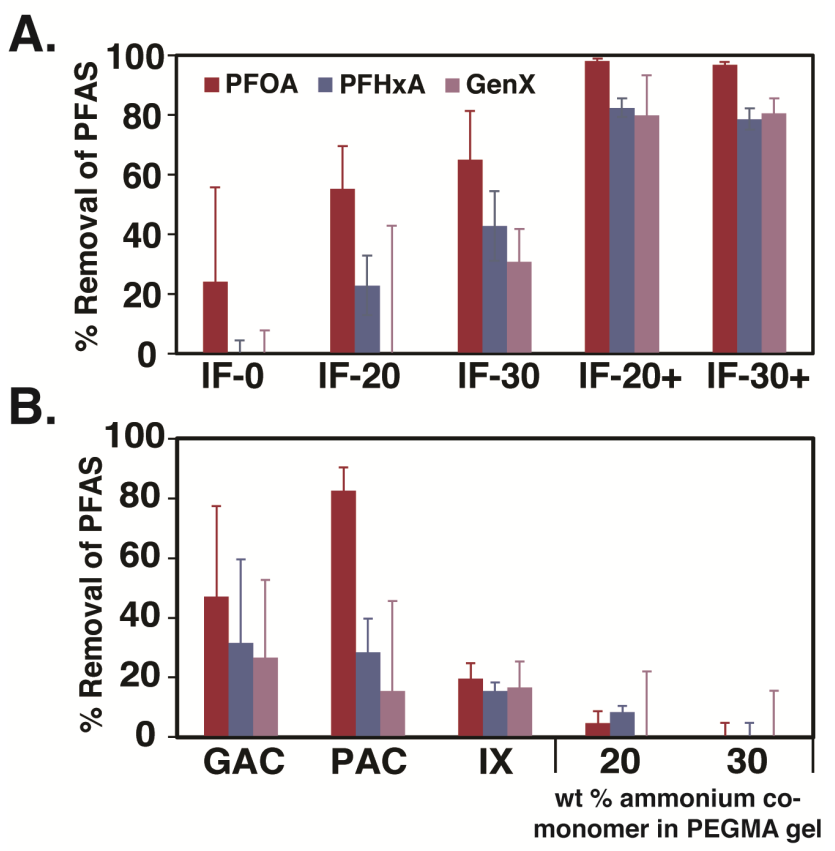

Figure 1: A. Equilibrium PFAS removal by Ionic Fluorogels with amine (IF-X) or ammonium (IF-X+) groups where $X=0,20$, or $30 \mathrm{wt} \%$. B. Equilibrium PFAS removal by GAC, Powdered Activated Carbon (PAC), Ion Exchange Resin (IX) and PEGMA gels with 20 or $30 \mathrm{wt} \%$ ammonium comonomer. Water constituents: $200 \mathrm{mg} / \mathrm{L} \mathrm{NaCl}$ and 20 $\mathrm{mg} / \mathrm{L}$ humic acid; $\mathrm{pH}=6.4$; Sorbent: $10 \mathrm{mg} / \mathrm{L}$; PFAS: (PFOA, PFHxA, GenX, $1 \mu \mathrm{g} \mathrm{L}^{-1}$ each); Equilibrium time: 21 h. Error bars: Standard deviation of 3 experiments.

Comparing Ionic Fluorogels against materials made to serve as controls illustrated the synergistic roles of fluorous interactions and ion exchange behavior. Exposing IF-0 (Figure 1A), an Ionic Fluorogel made solely of Fluorolink $^{\circledR}$, to the equilibrium adsorption experiment led to no removal of PFHxA or GenX. Furthermore, PEGMA gels made with a non-fluorous hydrocarbon equivalent of PFPEs demonstrated poor results for all formulations tested ( $<10 \%$ removal for all PFAS, Figure 1B).

Commercial materials representing the current state-ofthe-art for PFAS removal were subsequently tested under these equilibrium adsorption conditions. Samples of GAC (Filtrasorb 400, Calgon Carbon), powdered activated carbon (PAC, PicaHydro MP23, Lenntech) and an anion exchange resin (PFA 694E, Purolite) were exposed to simulated natural water for 21 hours at a resin loading of $10 \mathrm{mg} / \mathrm{L} \cdot{ }^{12,20,43}$ Under these challenging conditions, the limitations of current technology is evident, especially for the adsorption of short-chain PFAS (Figure 1B). These head-to-head comparisons demonstrate the selectivity of Ionic Fluorogels for PFAS compared to conventional technologies, especially in a complex matrix that contains a 20,000 times higher concentration of organic contaminants (humic acid) compared to each PFAS.

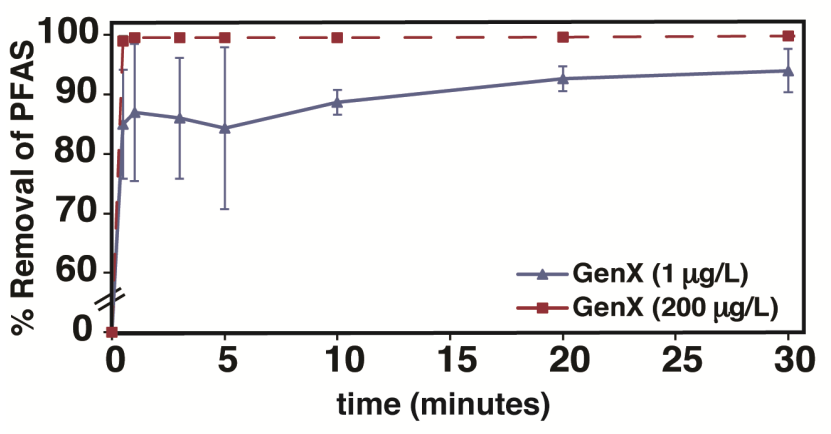

Figure 2: Time dependent GenX sorption by IF-20+ at high (red, dashed; GenX $=200 \mu \mathrm{g} / \mathrm{L}$; sorbent $=100 \mathrm{mg} / \mathrm{L}$ ) and low (blue, solid; GenX $=1 \mu \mathrm{g} / \mathrm{L}$; sorbent $=10 \mathrm{mg} / \mathrm{L}$ ) concentration. $\mathrm{pH}=9.7$. Error bars: Standard deviation of 3 experiments.

GenX was chosen as an emerging short-chain contaminant to understand the kinetics of adsorption and capacity of Ionic Fluorogels. IF-20+ and IF-30+ were chosen as high-performing materials for further study. The adsorption kinetics of GenX at high concentration (200 $\mu \mathrm{g} / \mathrm{L})$ by $\mathbf{I F - 2 0}+(100 \mathrm{mg} / \mathrm{L})$ were analyzed in deionized water (Figure 2). Rapid and quantitative removal of GenX was observed within 30 seconds. At 72 hours, no desorption was observed, suggesting the adsorption into the Ionic Fluorogel is irreversible under these conditions. Similarly, the adsorption kinetics at an environmentally relevant concentration of GenX $(1 \mu \mathrm{g} / \mathrm{L})$ by IF-20+ (10 mg/L) was also rapid, demonstrating 94\% removal within 30 minutes and no desorption over time (Figure 2). This removal efficiency for GenX results in a final concentration $(60 \mathrm{ng} / \mathrm{L})$, under the limit set by the state of North Carolina (140 ng/L).

A GenX binding isotherm was constructed to understand the binding capacity of IF-20+. ${ }^{44}$ The concentration of IF-20+ was fixed at $100 \mathrm{mg} / \mathrm{L}$ while the GenX concentration was varied from $0.20-50 \mathrm{mg} / \mathrm{L}$. Data from triplicate experiments (Figure 3A) was fit to the Langmuir adsorption model to yield an affinity coefficient $\left(\mathrm{K}_{\mathrm{L}}\right)$ of $5.9 \times 10^{6} \mathrm{M}^{-1}$ and an estimated GenX capacity $\left(\mathrm{Q}_{\mathrm{m}}\right)$ of $278 \mathrm{mg} / \mathrm{g}$. These represent the highest reported values in the literature for GenX. ${ }^{28}$ The isotherm was also fit to Freundlich model and the Freundlich's constant $\left(\mathrm{K}_{\mathrm{F}}\right)$ and the intensity of adsorption (n) were found to be 141 $(\mathrm{mg} / \mathrm{g})(\mathrm{L} / \mathrm{mg})^{1 / \mathrm{n}}$ and 2.2 respectively. $^{28}$

Subsequently, IF-20+ was tested for its ability to be regenerated for multiple reuse cycles (Figure 3B). Adsorption experiments were performed by loading IF-20+ (20 $\mathrm{mg})$ onto a PTFE syringe filter $(0.45 \mu \mathrm{m}, 25 \mathrm{~mm}$ diameter). A GenX solution (10 mg/L, $20 \mathrm{~mL})$ was passed through the filter over 2 minutes, and the residual GenX concentration in the filtrate was analyzed by LC-MS. The results showed $>90 \%$ removal of GenX from the solution in such flow-through conditions, thus demonstrating the efficiency of adsorption even under short 
residence time conditions. Complete extraction of adsorbed GenX by IF-20+ was achieved by washing the material with a $400 \mathrm{mM}$ methanolic ammonium acetate solution $(20 \mathrm{~mL}, 2 \mathrm{~min})$. This process was repeated 5 times without loss of efficiency in adsorption or reuse. A complementary analysis of IF-20+ regeneration under saturation conditions (Figure 3B inset and Figure S12) demonstrated that the resin is capable of regeneration and subsequent reuse under the high-capacity conditions relevant to water treatment.
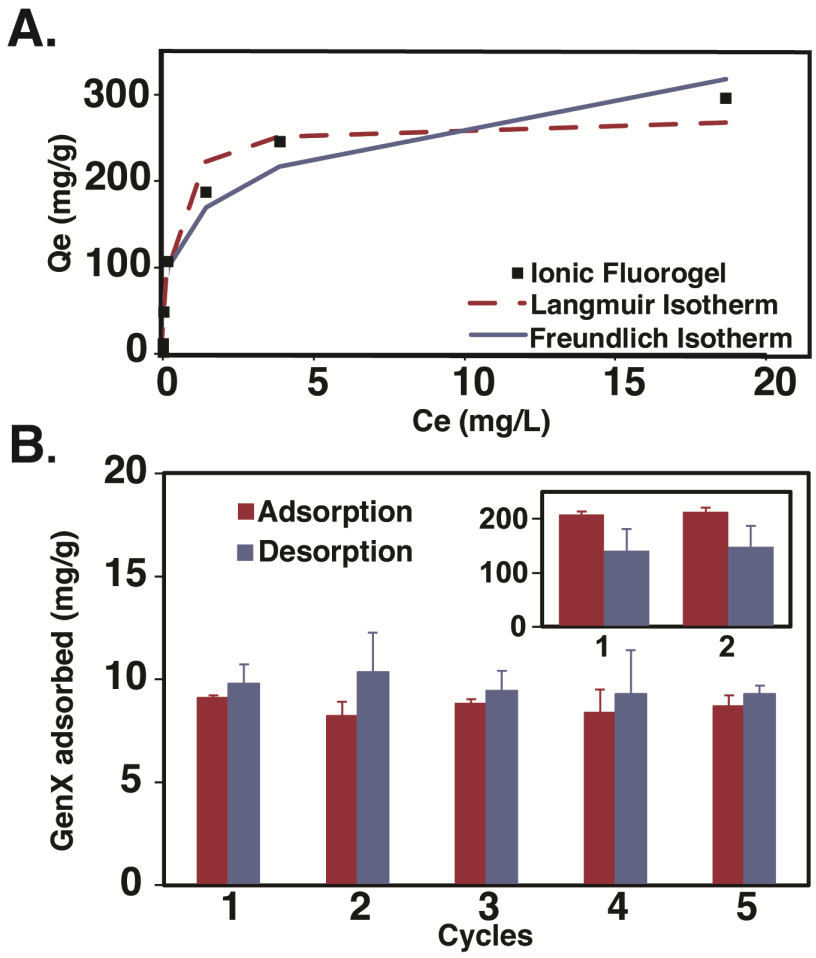

Figure 3: A. GenX adsorption isotherm by IF-20+ (Adsorbent $=100 \mathrm{mg} / \mathrm{L} ;$ GenX $=0.2-50 \mathrm{mg} / \mathrm{L})$. Lines show fit to Langmuir (red, dashed) and Freundlich (blue, solid) models. B. Regeneration and reuse of IF-20+. Sorbent $=20 \mathrm{mg}$, $[\mathrm{GenX}]=10 \mathrm{mg} / \mathrm{L}(20 \mathrm{~mL})$; extraction with $400 \mathrm{mM}$ methanolic ammonium acetate $(20 \mathrm{~mL})$. Inset: Sorbent $=5 \mathrm{mg}$, $[\mathrm{GenX}]=200 \mathrm{mg} / \mathrm{L}(5 \mathrm{~mL})$; extraction with $400 \mathrm{mM}$ methanolic ammonium acetate (5 $\mathrm{mL})$. Error bars: Standard deviation of 3 experiments.

The Ionic Fluorogels provided rapid, efficient, and high capacity removal of a variety of PFAS under laboratory conditions. Real water matrices, however, contain an unpredictable cocktail of organic and inorganic contaminants that are difficult to model in a laboratory setting. To validate Ionic Fluorogels as a promising technology for PFAS removal from water, we obtained settled water collected at a site previously affected by PFAS contamination, the Sweeney Water Treatment Plant in Wilmington, NC. ${ }^{12}$ The water contained a total organic content (TOC) of $1.3 \mathrm{mg} / \mathrm{L}$ and had a $\mathrm{pH}$ of 6.2 . In addition to PFAS found in the water upon collection (at levels of 20-50 ng/L), we spiked the matrix with 21 emerging and legacy PFAS. The real water matrix was exposed to IF30+ $(100 \mathrm{mg} / \mathrm{L})$, and PFAS removal was analyzed at 30 minutes and 2 hours, with the data presented being the average of two experiments. After 2 hours, short chain PFAS that are traditionally challenging to adsorb, including PFHxA, GenX, and PFBS are removed from the water at $>95 \%$ efficiency. We did not see evidence of long chain PFAS such as PFOA and PFOS in the solution down to the detection limit of the LC-MS (representative data shown in Figure 4, complete analysis in Figure S11). Lastly, IF-30+ performed impressively for removing the short chain perfluorinated carboxylic acids PFBA (60\%) and PFPeA (87\%), which contain only 3 and 4 perfluorocarbons, respectively (Figure 4). ${ }^{27,28,32}$

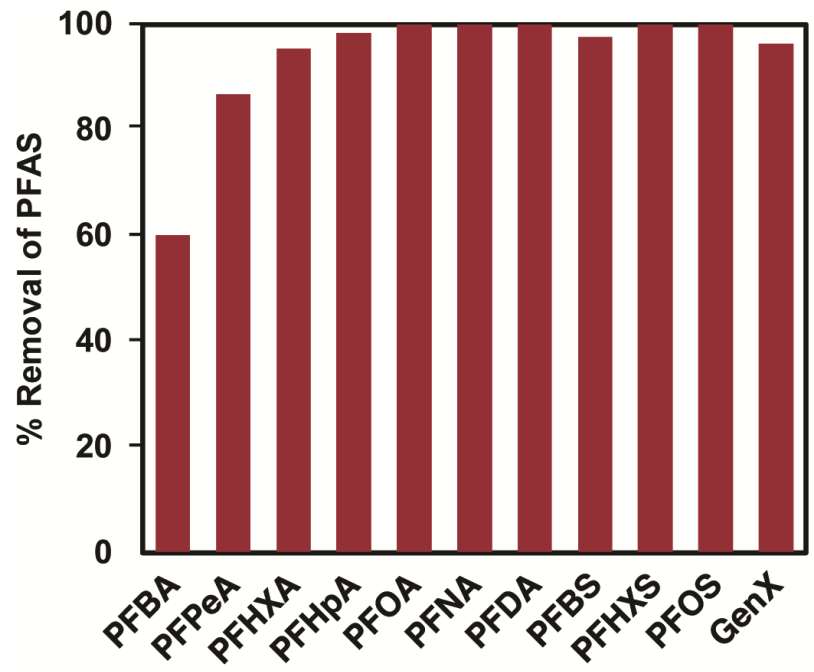

Figure 4: Removal of PFAS after 2 hours by IF-30+ from settled water collected at the Sweeney Water Treatment Plant in Wilmington, NC. TOC $=1.3 \mathrm{mg} / \mathrm{L}$; $\mathrm{pH}=6.2 ;[$ Adsorbent $]=100 \mathrm{mg} / \mathrm{L} ;[\text { PFAS }]_{0}=1 \mu \mathrm{g} / \mathrm{L}$ each. 10 Representative PFAS shown, complete analysis shown in Figure S11.

In conclusion, we introduce Ionic Fluorogels as a platform polymeric adsorbent to remove PFAS from water at environmentally relevant concentrations. The synergistic combination of fluorous and electrostatic interactions results in the high affinity, high capacity, and rapid sorption of a variety of PFAS from real water collected at an affected site in the Cape Fear River Watershed of North Carolina. The systematic material library and relationships identified between fluoropolymer and ionic content suggest general structure-property criteria to design improved sorbents for PFAS.

\section{ASSOCIATED CONTENT}

Supporting Information.

The Supporting Information is available free of charge on the ACS Publications website.

Experimental procedures and details (PDF) 


\section{AUTHOR INFORMATION}

\section{Corresponding Author}

* Frank1@email.unc.edu \& coronell@unc.edu

\section{Funding Sources}

This research was supported by the North Carolina Policy Collaboratory from funding appropriated by the North Carolina General Assembly. Instrumentation used in this publication was supported by the National Institute of Environmental Health Sciences of the National Institutes of Health under award number P30ES010126. The content is solely the responsibility of the authors and does not necessarily represent the official views of the National Institutes of Health.

\section{Notes}

\#: These authors contributed equally to this work

The authors declare the following competing financial interest(s): The University of North Carolina at Chapel Hill has filed for intellectual property protection based on these findings.

\section{ACKNOWLEDGMENTS}

We acknowledge the members of the North Carolina PFAS Testing Network for insightful discussions and support surrounding PFAS in North Carolina.

\section{REFERENCES}

Banks, R.E.; Smart, B.E.; Tatlow, J. C. Organofluorine Chemistry: Priciples and Comercial Applications; Springer US: Boston, MA, 1994.

Knepper, T. P.; Lange, F. T. Polyfluorinated Chemicals and Transformation Products; Springer Science \& Business Media, 2011; Vol. 17.

Kotthoff, M.; Müller, J.; Jürling, H.; Schlummer, M.; Fiedler, D. Perfluoroalkyl and Polyfluoroalkyl Substances in Consumer Products. Environ. Sci. Pollut. Res. 2015, 22 (19), 14546-14559.

Lindstrom, A. B.; Strynar, M. J.; Libelo, E. L. Polyfluorinated Compounds: Past, Present, and Future. Environ. Sci. Technol. 2011, 45 (19), 7954 7961.

Hu, X. C.; Andrews, D. Q.; Lindstrom, A. B.; Bruton, T. A.; Schaider, L. A.; Grandjean, P.; Lohmann, R.; Carignan, C. C.; Blum, A.; Balan, S. A.; et al. Detection of Poly- and Perfluoroalkyl Substances (PFASs) in U.S. Drinking Water Linked to Industrial Sites, Military Fire Training Areas, and Wastewater Treatment Plants. Environ.

Darrow, L. A.; Groth, A. C.; Winquist, A.; Shin, H.-M.; Bartell, S. M.; Steenland, K. Modeled Perfluorooctanoic Acid (PFOA) Exposure and Liver Function in a Mid-Ohio Valley Community. Sci. Technol. Lett. 2016, 3 (10), 344-350.

Environ. Health Perspect. 2016, 124 (8), 1227 1233.

Strynar, M.; Dagnino, S.; McMahen, R.; Liang, S.; Lindstrom, A.; Andersen, E.; McMillan, L.; Thurman, M.; Ferrer, I.; Ball, C. Identification of Novel Perfluoroalkyl Ether Carboxylic Acids (PFECAs) and Sulfonic Acids (PFESAs) in Natural Waters Using Accurate Mass Time-of-Flight Mass Spectrometry (TOFMS). Environ. Sci. Technol. 2015, 49 (19), 11622-11630. 
(17) Mccord, J.; Strynar, M. Identification of Per- and Polyfluoroalkyl Substances in the Cape Fear River by High Resolution Mass Spectrometry and Nontargeted Screening. Environ. Sci. Technol. 2019, 53 (9), 4717-4727.

(18) Yu, J.; Lv, L.; Lan, P.; Zhang, S.; Pan, B.; Zhang, W. Effect of Effluent Organic Matter on the Adsorption of Perfluorinated Compounds onto Activated Carbon. J. Hazard. Mater. 2012, 226, 99-106.

(19) Dudley, L.A., Arevalo, E.C., Knappe, D. R. U. Removal of Perfluoroalkyl Substances by PAC Adsorption and Anion Exchange; Water Research Foundation: Denver, Colorado, 2015.

(20) Ross, I.; McDonough, J.; Miles, J.; Storch, P.; Thelakkat Kochunarayanan, P.; Kalve, E.; Hurst, J.; S. Dasgupta, S.; Burdick, J. A Review of Emerging Technologies for Remediation of PFASs. Remediat. J. 2018, 28 (2), 101-126.

(21) Hopkins, Z. R.; Sun, M.; DeWitt, J. C.; Knappe, D. R. U. Recently Detected Drinking Water Contaminants: GenX and Other Per- and Polyfluoroalkyl Ether Acids. J. Am. Water Works Assoc. 2018, 110 (7), 13-28.

(22) Schachtman, B. CFPUA Filtered Water Still Has More Contaminants than Raw Water. Here's Why, and What's Being Done. Port City Daily. Wilmington, NC 2019.

(23) Alsbaiee, A.; Smith, B. J.; Xiao, L.; Ling, Y.; Helbling, D. E.; Dichtel, W. R. Rapid Removal of Organic Micropollutants from Water by a Porous $\beta$-Cyclodextrin Polymer. Nature 2016, 529 (7585), 190-194.

(24) Xiao, L.; Ling, Y.; Alsbaiee, A.; Li, C.; Helbling, D. E.; Dichtel, W. R. $\beta$ - Cyclodextrin Polymer Network Sequesters Perfluorooctanoic Acid at Environmentally Relevant Concentrations. $J$. Am. Chem. Soc. 2017, 139, 7680-7692.

(25) Ling, Y.; Klemes, M. J.; Xiao, L.; Alsbaiee, A.; Dichtel, W. R.; Helbling, D. E. Benchmarking Micropollutant Removal by Activated Carbon and Porous $\beta$ - Cyclodextrin Polymers under Environmentally Relevant Scenarios. Environ. Sci. Technol. 2017, 51, 7590-7598.

(26) Xiao, L.; Ching, C.; Ling, Y.; Nasiri, M.; Klemes, M. J.; Reineke, T. M.; Helbling, D. E.; Dichtel, W. R. Cross-Linker Chemistry Determines the Uptake Potential of Perfluorinated Alkyl Substances by $\beta$ Cyclodextrin Polymers. Macromolecules 2019, 52, 3747-3752.

(27) Klemes, A. M. J.; Ling, Y.; Ching, C.; Wu, V.; Helbling, E.; Dichtel, W. R. Reduction of a Tetrafluoroterephthalonitrile- $\beta$-Cyclodextrin Polymer to Remove Anionic Micropollutants and Perfluorinated Alkyl Substances from Water. Angew. Chem. Int. Ed. 2019, 58, 12049-12053.

Ji, W.; Xiao, L.; Ling, Y.; Ching, C.; Matsumoto,
M.; Bisbey, R. P.; Helbling, D. E.; Dichtel, W. R. Removal of GenX and Perfluorinated Alkyl Substances from Water by Amine-Functionalized Covalent Organic Frameworks. J. Am. Chem. Soc. 2018, 140 (40), 12677-12681.

Cao, F.; Wang, L.; Ren, X.; Sun, H. Synthesis of a Perfluorooctanoic Acid Molecularly Imprinted Polymer for the Selective Removal of Perfluorooctanoic Acid in an Aqueous Environment. J. Appl. Polym. Sci. 2016, 133 (15), 1-10.

(30) Yu, Q.; Deng, S.; Yu, G. Selective Removal of Perfluorooctane Sulfonate from Aqueous Solution Using Chitosan-Based Molecularly Imprinted Polymer Adsorbents. Water Res. 2008, 42, 30893097.

(31) Huang, P. J.; Hwangbo, M.; Chen, Z.; Liu, Y.; Kameoka, J.; Chu, K. H. Reusable Functionalized Hydrogel Sorbents for Removing Long- and ShortChain Perfluoroalkyl Acids (PFAAs) and GenX from Aqueous Solution. ACS Omega 2018, 3 (12), 17447-17455.

(32) Ateia, M.; Attia, M. F.; Maroli, A.; Tharayil, N.; Alexis, F.; Whitehead, D. C.; Karanfil, T. Rapid Removal of Poly- and Perfluorinated Alkyl Substances by Poly(Ethylenimine)-Functionalized Cellulose Microcrystals at Environmentally Relevant Conditions. Environ. Sci. Technol. Lett. 2018, 5 (12), 764-769.

(33) Koda, Y.; Terashima, T.; Sawamoto, M. Fluorous Microgel Star Polymers: Selective Recognition and Separation of Polyfluorinated Surfactants and Compounds in Water. J. Am. Chem. Soc. 2014, 136, 15742-15748.

(34) Koda, Y.; Terashima, T.; Takenaka, M.; Sawamoto, M. Star Polymer Gels with Fluorinated Microgels via Star-Star Coupling and CrossLinking for Water Purification. ACS Macro Lett. 2015, 4 (4), 377-380.

(35) Zhang, W.; Curran, D. P. Synthetic Applications of Fluorous Solid-Phase Extraction (F-SPE). Tetrahedron 2006, 62 (51), 11837-11865.

(36) Luo, Z.; Zhang, Q.; Oderaotoshi, Y.; Curran, D. P. Fluorous Mixture Synthesis: A Fluorous-Tagging Strategy for the Synthesis and Separation of Mixtures of Organic Compounds. Science (80-. ). 2001, 291 (5509), 1766-1769.

(37) Curran, D. P. Strategy-Level Separations in Organic Synthesis: From Planning to Practice. Angew. Chem. Int. Ed. 1998, 37 (9), 1174-1196.

(38) Bell, G. A.; Howell, J. Perfluoropolyethers. In Synthetics, Mineral Oils, and Bio-Based Lubricants: Chemistry and Technology; Rudnick, L. R., Ed.; CRC PRess: Boca Raton, FL, 2005; pp 157-174.

(39) Howell, J. L.; Perez, E. W.; Waterfeld, A.; Friesen, C. M.; Thrasher, J. S. Thermally Stable 
Perfluoropolyethers and Processes Therefore and Therewith. US6753301B2, 2001.

(40) Faucitano, A.; Buttafava, A.; Commincioli, V.; Marchionni, G.; Pasquale, R. J. D. E. Kinetic Modeling of the Low-Temperature PhotoOxidation of Hexafluoropropene. J. Phys. Org. Chem. 1991, 4 (June 1990), 293-300.

(41) Bunyard, W. C.; Romack, T. J.; Desimone, J. M. Perfluoropolyether Synthesis in Liquid Carbon Dioxide by Hexafluoropropylene Photooxidation. Macromolecules 1999, 32 (24), 8224-8226.

(42) Kasai, P. H. Perfluoropolyethers: Intramolecular
Disproportionation. Macromolecules 1992, 25 (25), 6791-6799.

(43) Zaggia, A.; Conte, L.; Falletti, L.; Fant, M.; Chiorboli, A. Use of Strong Anion Exchange Resins for the Removal of Perfluoroalkylated Substances from Contaminated Drinking Water in Batch and Continuous Pilot Plants. Water Res. 2016, 91, 137-146.

(44) Bolster, C. H.; Green, B.; Hornberger, G. M. On the Use of Linearized Langmuir Equations. Soil Sci. Soc. Am. J. 2007, 71 (6), 1796-1806. 


\section{Ionic Fluorogels for Remediation of Per- and Polyfluorinated Alkyl Substances from Water}

Elango Kumarasamy, ${ }^{1, \#}$ Irene M. Manning, ${ }^{1, \#}$ Leonard B. Collins, ${ }^{2}$ Orlando Coronell, ${ }^{2}$ and Frank A. Leibfarth*1

1'Department of Chemistry, University of North Carolina at Chapel Hill, Chapel Hill, NC, 27599, USA

${ }^{2}$ Department of Environmental Sciences and Engineering, Gillings School of Global Public Health, University of North Carolina at Chapel Hill, Chapel Hill, NC 27599, USA

\#These authors contributed equally to this work

\section{TABLE OF CONTENTS}

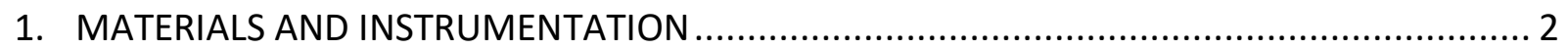

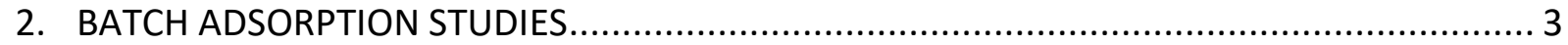

2.1.Equilibrium adsorption studies .................................................................... 3

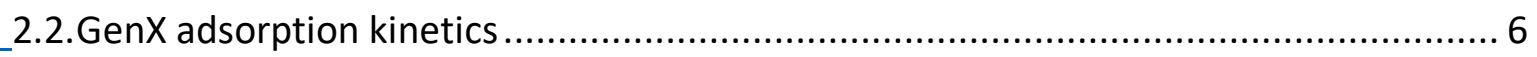

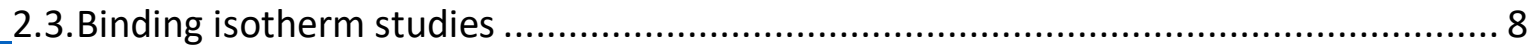

2.4. Real water studies

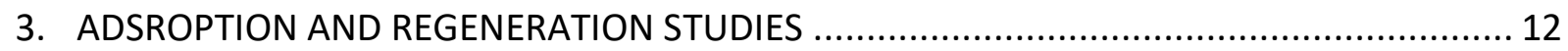

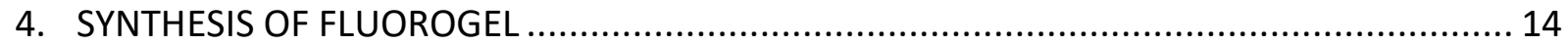

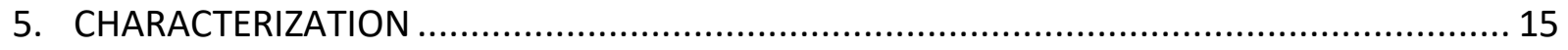

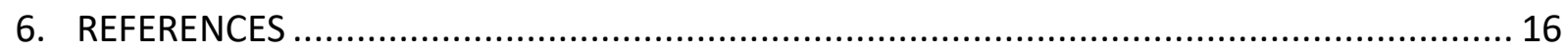




\section{MATERIALS AND INSTRUMENTATION}

Materials: All materials were purchased from commercial source and used as received without further purification unless otherwise mentioned. Perfluoropolyether Fluorolink ${ }^{\circledR}$ MD700 (Mwt: 1.8-2.0 kg. mol $^{-1}$ ) was purchased from Solvay Solexis. 2-(dimethylamino)ethyl methacrylate, Poly(ethylene glycol) dimethacrylate (average $M n$ 750), azobisisobutyronitrile (AIBN), humic acid and perfluorooctanoic acid (PFOA) were purchased from Sigma-Aldrich. Trifluoroethanol was purchased Synquest labs. Perfluorohexanoic acid (PFHXA) and GenX were purchased from TCl and Matrix respectively.

\section{Instrumentation:}

LCMS: Water samples were stored under refrigeration until analysis. A $196 \mu \mathrm{L}$ aliquot of sample and $4 \mu \mathrm{L}$ of stable isotope-labeled analogues (Wellington Labs, Guelph, CA, product numbers MPFAC-C-ES and M3HFPO-DA) were transferred to polypropylene autosampler vials and closed with caps fitted with silicone septa. No other processing was done as per a direct injection method by M. Sun et al. ${ }^{1}$

Analysis of target compounds was performed using an Accela HPLC system coupled to a TSQ-Quantum Ultra triple-quadrupole mass analyzer (Thermo Scientific, San Jose, CA) operated in negative ion mode. Samples were chromatographed on a $3.0 \times 50 \mathrm{~mm}$ Poroshell C18 $2.7 \mu \mathrm{m}$ column (Agilent Technologies, Santa Clara, CA) with gradient elution at a flow rate of $350 \mu \mathrm{L}$ per min. Binary mobile phase consisted of 95:5:water:methanol containing $2 \mathrm{mM}$ ammonium acetate $(\mathrm{A})$ and 5:95:water:methanol containing $2 \mathrm{mM}$ ammonium acetate (B). Composition started at $25 \%$ B, was held for $0.5 \mathrm{~min}$., increased linearly to $90 \%$ B over 2 min., was held at $90 \%$ B for 1.5 min., decreased linearly to $25 \%$ B over 0.1 min., and held at $25 \%$ B for 0.9 min for column equilibration. Mass spectrometer parameters were as follows: spray voltage of 3000 $\mathrm{V}$, vaporizer temperature of $150^{\circ} \mathrm{C}$, sheath gas flow rate 40 , auxiliary gas flow rate 20 , capillary temperature of $225^{\circ} \mathrm{C}$, argon collision gas pressure of $1.0 \mathrm{mTorr}, 0.05 \mathrm{sec}$ per scan, quadrupole 1 resolution of $0.5 \mathrm{amu}$, quadrupole 3 resolution of 0.7 amu and collision energy $10 \mathrm{eV}$. Mass transitions and other compoundspecific parameters are listed in Table 1. The limit of detection was 2 pg per $100 \mu \mathrm{L}(20 \mathrm{pg} / \mathrm{mL})$ injection for each analyte. Linear or quadratic calibration curves using the analyte to internal standard ratio were used to calculate analyte amounts. Calibration points were 2, 10, 50, 200, and $1000 \mathrm{pg}$ analyte versus 50 pg internal standard for PFCAs and PFASs.

Thermal gravimetric analysis (TGA) was performed on a TA Instruments TGA (Discovery Series) using 5$8 \mathrm{mg}$ of the sample. The samples were heated to $25-600{ }^{\circ} \mathrm{C}$ at a temperature ramp rate of $10{ }^{\circ} \mathrm{C} / \mathrm{min}$. Infrared (IR) spectra were obtained using PerkinElmer Frontier FT-IR spectrometer.

Deionized water used in this study is a type 1, 18.2 megohm-cm water obtained from Labconco - waterpro PS series. This water was amended with sodium chloride and humic acid if necessary.

Centrifugation was performed using a benchtop centrifuge - Mini mouse II by Denville. 


\section{BATCH ADSORPTION STUDIES}

\subsection{Equilibrium adsorption studies}

The batch adsorption studies of mixtures of PFAS (PFOA, PFHxA and GenX) was performed in a $1 \mathrm{~L}$ HDPE bottle equipped with a magnetic stir bar. The mixture was stirred on a stir plate at room temperature and at 700 revolution per minute (rpm).

High Concentration (50 $\mathrm{gg} / \mathrm{L})$ : To a $1 \mathrm{~L}$ deionized water added sodium chloride $(200 \mathrm{mg})$ and humic acid (20 mg) and stirred overnight. To this mixture added vacuum dried polymer adsorbent (ionic fluorogel) (10 $\mathrm{mg} \mathrm{L}^{-1}$ ) and stirred at room temperature for $3 \mathrm{~h}$ with occasional sonication to disperse the adsorbent. A stock solution of PFAS was spiked to the mixture to create an initial concentration of $50 \mu \mathrm{g} \mathrm{L}^{-1}$ of each PFAS. This mixture was stirred for $21 \mathrm{~h}$ after which an aliquot of about $10 \mathrm{~mL}$ was withdrawn and filtered through either $0.2 \mu \mathrm{m}$ PTFE or $0.45 \mu \mathrm{m}$ cellulose acetate filter. The first $5 \mathrm{~mL}$ was drained to avoid any electrostatic effect from the filter and the remaining $5 \mathrm{~mL}$ was collected for LCMS analysis. Control experiments to account for PFAS losses during handling were performed under identical condition in the absence of adsorbent. This batch experiment was performed once due to the high adsorption observed.

Low Concentration (1 $\mathrm{g} / \mathrm{L})$ : The batch adsorption studies of PFAS under environmentally relevant concentration ( $1 \mu \mathrm{g} \mathrm{L}^{-1}$ ) was performed under identical condition as detailed above except that the PFAS was spiked to create an initial concentration of $1 \mu \mathrm{g} \mathrm{L}^{-1}$ of each PFAS. This set of experiments were performed in triplicates.

Control adsorbents used in the study granular activated carbon (GAC: Filtrasorb 400), powdered activated carbon (PAC: PicaHydro MP23) and ion-exchange resin (IX: PFA694E) were purchased from commercial sources.

The efficiency of PFAS removal by adsorbents was determined by the following equation

$$
\text { \% PFAS removal }=\frac{C_{0}-C_{t}}{C_{0}} \times 100 \begin{aligned}
& C_{0}\left(\mu \mathrm{g} \mathrm{L}^{-1}\right)=\text { Initial concentration of PFAS } \\
& C_{t}\left(\mu \mathrm{g} \mathrm{L}^{-1}\right)=\text { Residual concentration of PFAS }
\end{aligned}
$$

The amount of PFAS bound to the polymer sorbent is given by the following equation

$$
\begin{array}{ll}
q_{t}\left(\mathrm{mg} \mathrm{g}^{-1}\right)=\text { Amount of PFAS adsorbed on the solid phase at time } t(\mathrm{~h}) \\
C_{t}\left(\mu \mathrm{g} \mathrm{L}^{-1}\right)=\text { Concentration of PFAS in liquid phase at time } t(\mathrm{~h}) \\
C_{o}\left(\mu \mathrm{g} \mathrm{L}^{-1}\right)=\text { Average concentration of PFAS in control experiments } \\
C_{A}\left(\mathrm{mg} \mathrm{L}^{-1}\right)=\text { Concentration of adsorbent }
\end{array}
$$




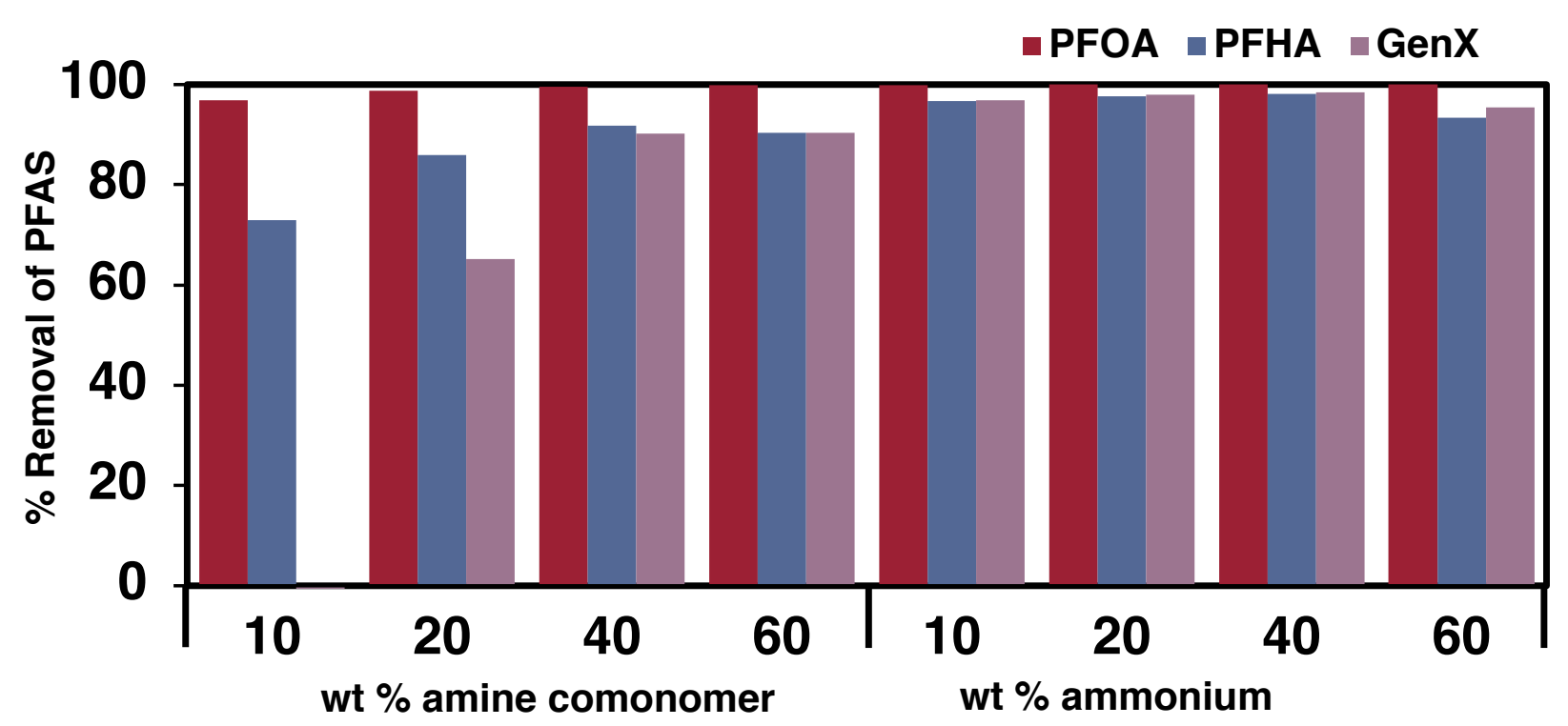

Figure S1: Equilibrium PFAS removal efficiency of different compositions of lonic Fluorogel in presence of $\mathrm{NaCl}(200 \mathrm{ppm})$ and humic acid (20 ppm). PFAS: PFOA, PFHxA and GenX (each $50 \mu \mathrm{g} / \mathrm{L})$. adsorbent dosage: $100 \mathrm{mg} / \mathrm{L}$. equilibrium time: $21 \mathrm{~h}$.

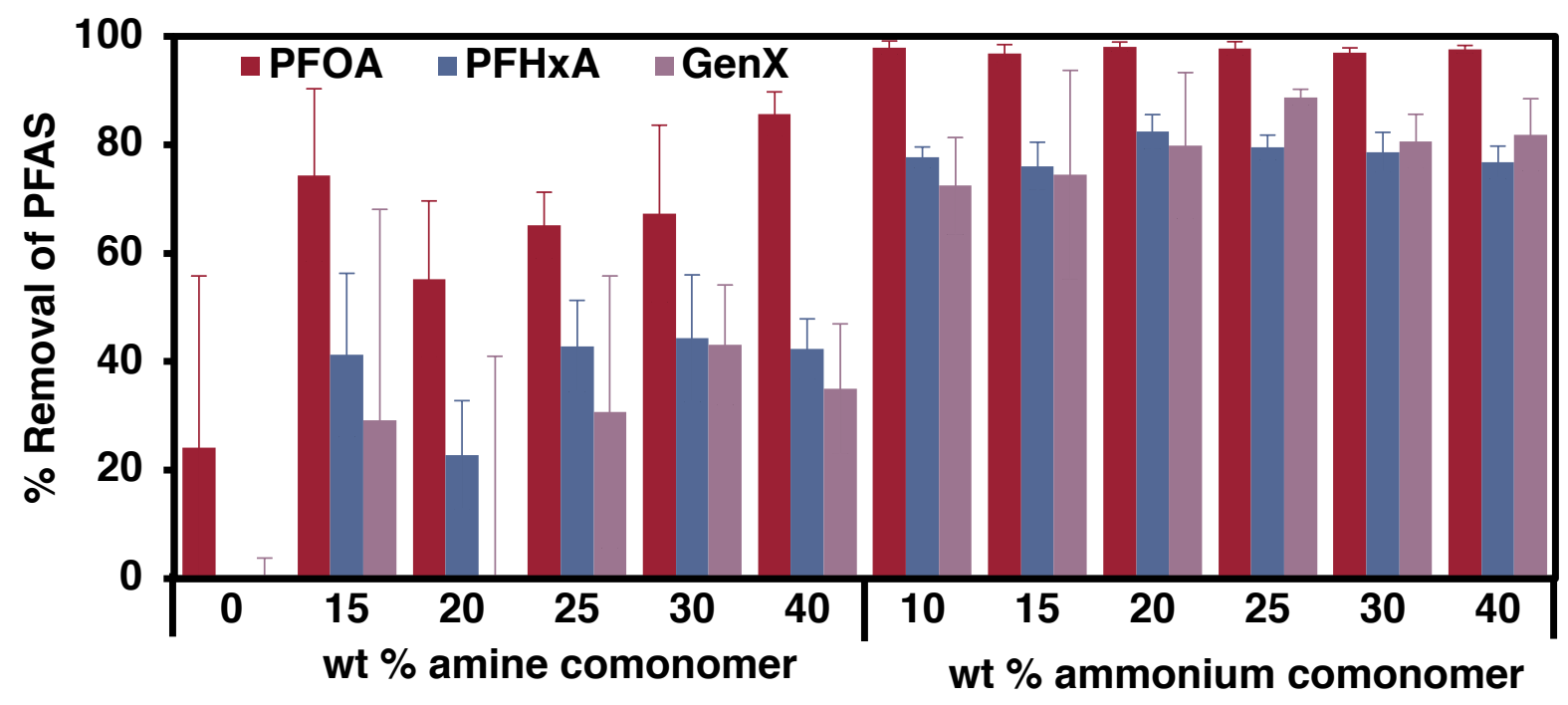

Figure S2: Equilibrium PFAS removal efficiency by various compositions of lonic Fluorogels in presence of $\mathrm{NaCl}(200 \mathrm{ppm})$ and humic acid (20 ppm). PFAS: PFOA, PFHxA and GenX (each $1 \mu \mathrm{g} / \mathrm{L})$. adsorbent dosage: $10 \mathrm{mg} / \mathrm{L}$. equilibrium time: $21 \mathrm{~h}$. The data points in the figure are an average of 3 experiments and the error bar show their standard deviation. 


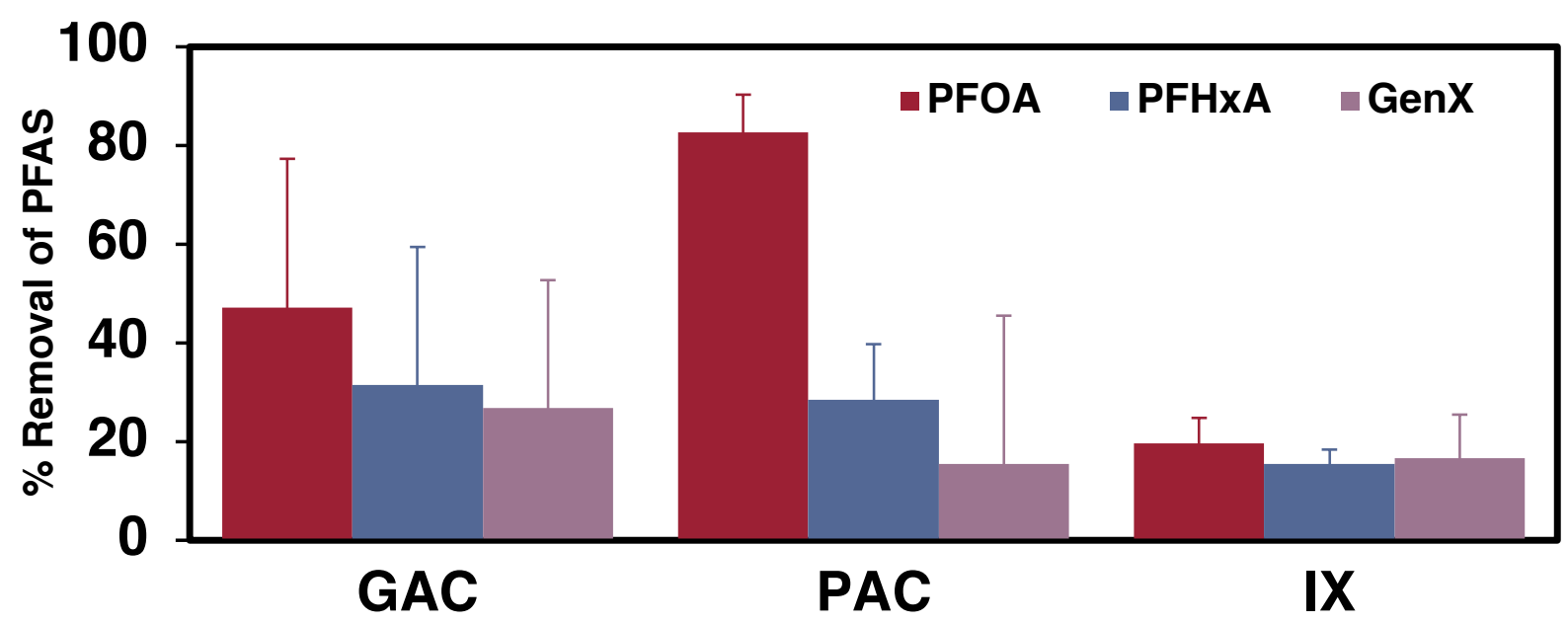

Figure S3: Equilibrium PFAS removal efficiency by granular activated carbon(GAC), powdered activated carbon (PAC) and ion-exchange resin (IX), in presence of $\mathrm{NaCl}(200 \mathrm{ppm})$ and humic acid (20 ppm). PFAS: PFOA, PFHXA and GenX (each $1 \mu \mathrm{g} / \mathrm{L}$ ). adsorbent dosage: $10 \mathrm{mg} / \mathrm{L}$. equilibrium time: $21 \mathrm{~h}$. The data points in the figure are an average of 3 experiments and the error bar show their standard deviation.

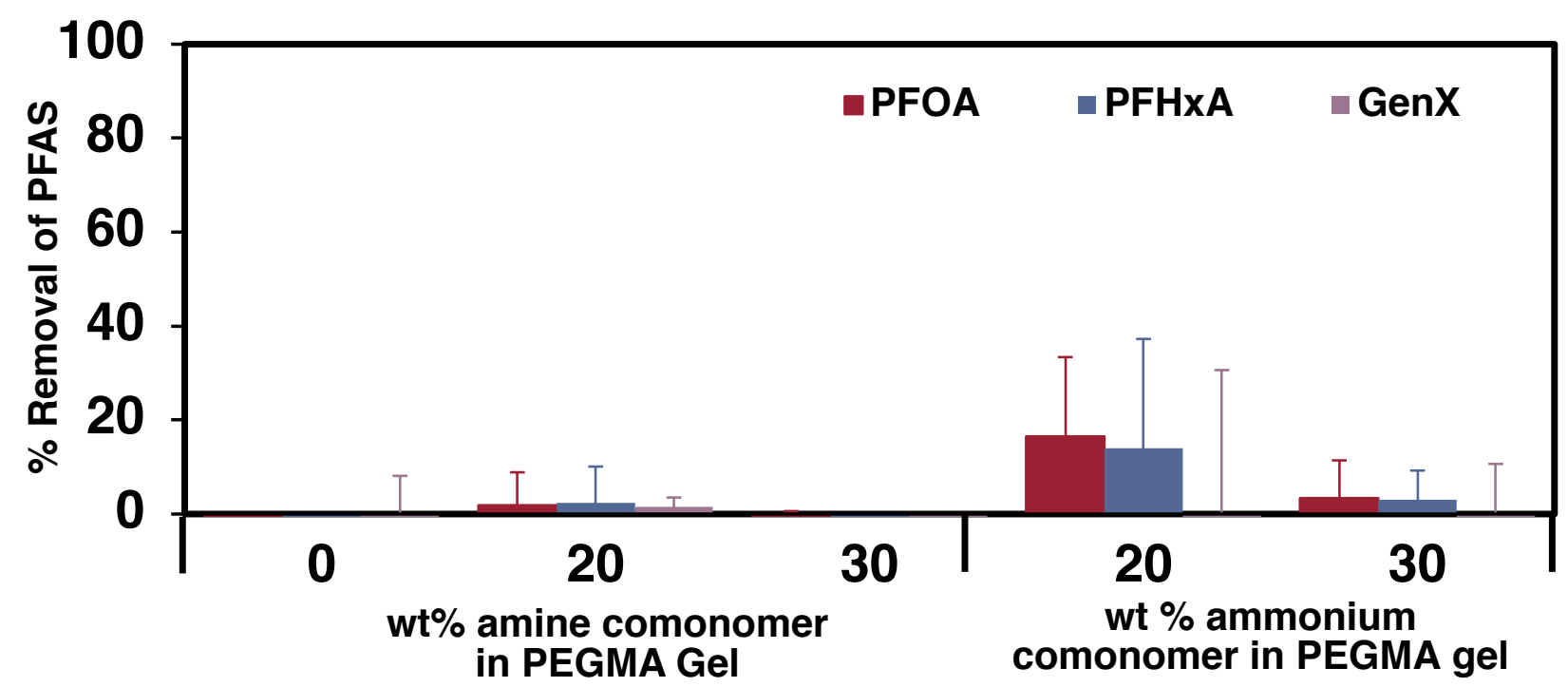

Figure S4: Equilibrium PFAS removal efficiency by gel made from polyethylene glycol diacrylate (PEGDA, $\mathrm{Mn} 750$ ) in presence of $\mathrm{NaCl}(200$ ppm) and humic acid (20 ppm). PFAS: PFOA, PFHxA and GenX (each $1 \mu \mathrm{g} / \mathrm{L})$. adsorbent dosage: $10 \mathrm{mg} / \mathrm{L}$. equilibrium time: $21 \mathrm{~h}$. 


\subsection{GenX adsorption kinetics}

High concentration $(200 \mu g / L)$ : The adsorption kinetic experiments were performed in $125 \mathrm{~mL}$ polypropylene bottle equipped with a magnetic stir bar. The experiments were performed at room temperature on a multi-position stirrer at $500 \mathrm{rpm}$. The adsorbent dose was set at $10 \mathrm{mg} / \mathrm{L}$ with total operating volume of $100 \mathrm{~mL}$. The fluorogel and water mixture was stirred for $3 \mathrm{~h}$ with occasional sonication to disperse the adsorbent before being spiked with GenX stock to create an initial concentration of 200 $\mu \mathrm{g} / \mathrm{L}$. About $1 \mathrm{~mL}$ aliquot was taken at each predetermined time intervals $(0.5,1,5,10,30,60$, mins and 21,48 and $72 \mathrm{~h}$ ). The aliquots were centrifuged for 15 minutes and the supernatant was analyzed by LCMS to determine the residual GenX concentration. Control experiments to account for GenX losses during handling were performed under identical condition in the absence of adsorbent. This batch kinetics experiment was performed in triplicates.

Low concentration (1 $\mu \mathrm{g} / \mathrm{L}$ ): About $5 \mathrm{mg}$ of lonic fluorogel was taken in an $8 \mathrm{~mL}$ vial, followed by addition of DI water to create a concentration of $1 \mathrm{mg} / \mathrm{mL}$. The mixture was subjected to series of vortex and sonication to completely disperse ionic fluorogel. $1 \mathrm{~mL}$ of this mixture was taken while under constant mixing and added to $99 \mathrm{~mL}$ of water in a polypropylene bottle $(125 \mathrm{~mL})$ equipped with a magnetic stir bar. The mixture was stirred at $500 \mathrm{rpm}$ for $3 \mathrm{~h}$ before being spiked with GenX stock to create an initial concentration of $1 \mu \mathrm{g} / \mathrm{L}$. About $1 \mathrm{~mL}$ aliquot was taken at each predetermined time intervals $(0.5,1,3,5,10,20,30,60$, 120, 240 mins and 21, 48 and $72 \mathrm{~h}$ ). The aliquots were centrifuged for 15 minutes and the supernatant was analyzed by LCMS to determine the residual GenX concentration. Control experiments to account for GenX losses during handling were performed under identical condition in the absence of adsorbent. This batch kinetics experiment was performed in triplicates.

The kinetics of adsorption can be described with Ho and McKay's linearized form of pseudo-second-order adsorption model given by following equation ${ }^{2}$

$$
\begin{array}{ll}
\frac{t}{q_{t}}=\frac{t}{q_{e}}+\frac{1}{k_{o b s} q_{e}^{2}} & \begin{array}{l}
q_{e}\left(\mathrm{mg} \mathrm{g}^{-1}\right)=\text { Amount of GenX adsorbed on the solid phase at equilibrium } \\
k_{o b s}\left(\mathrm{~g} \mathrm{mg}^{-1} \mathrm{~h}^{-1}\right)=\text { Rate of adsorption } \\
q_{t}\left(\mathrm{mg} \mathrm{g}^{-1}\right)=\text { Amount of GenX adsorbed on the solid phase at time } t(\mathrm{~h})
\end{array}
\end{array}
$$



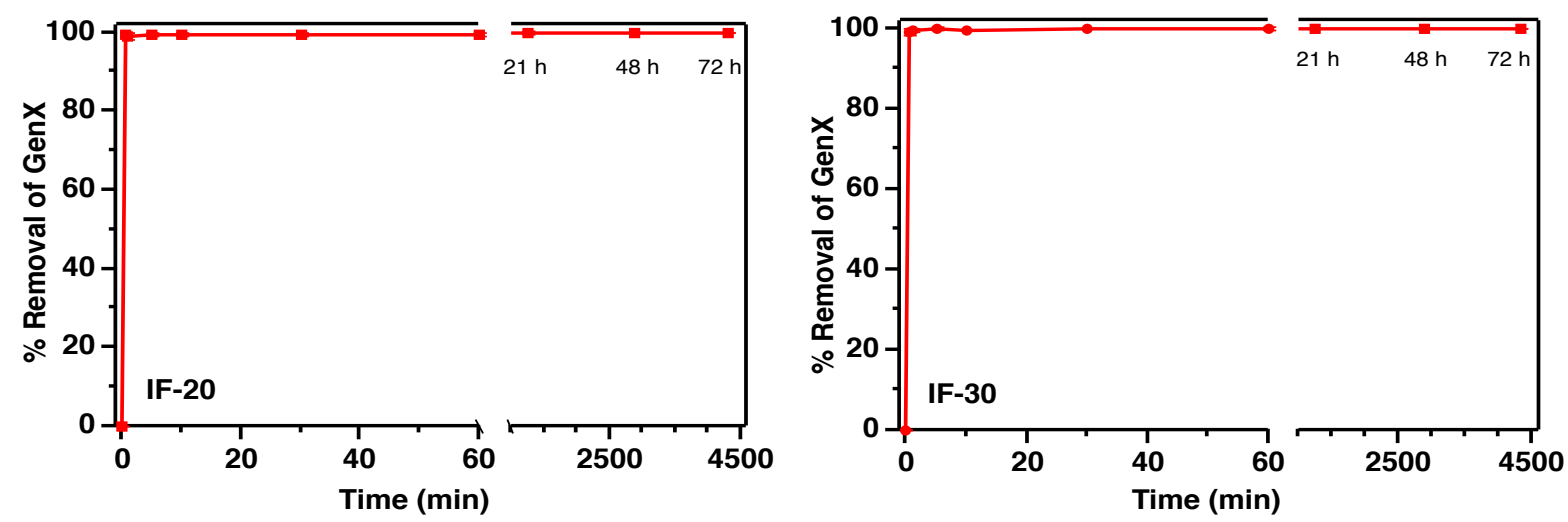

Figure S5: Kinetics of GenX (200 $\mu \mathrm{g} / \mathrm{L})$ adsorption by lonic Fluorogel with 20 wt $\%$ quaternized DMEAMA (left) and lonic Fluorogel with $30 \mathrm{wt} \%$ quaternized DMEAMA (right). Adsorbent dosage: $100 \mathrm{mg} / \mathrm{L}$. The data points in the figure are an average of 3 experiments and the error bar show their standard deviation.
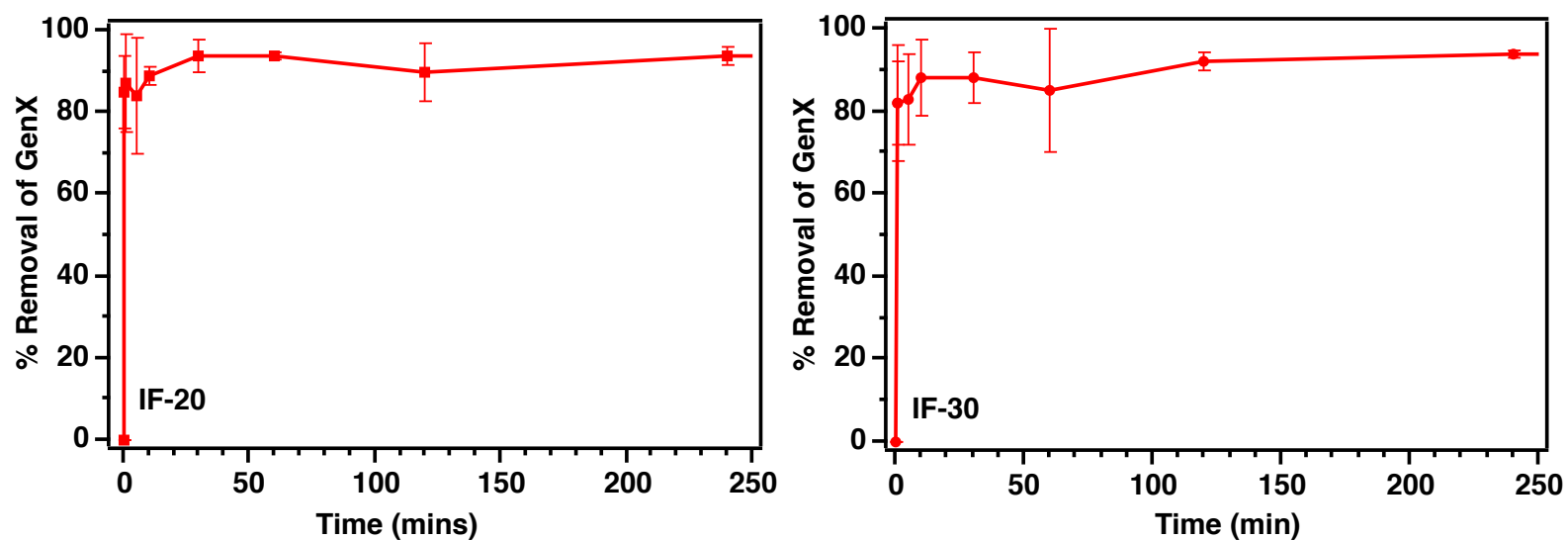

Figure S6: Kinetics of GenX $(1 \mu \mathrm{g} / \mathrm{L})$ adsorption by lonic Fluorogel with 20 wt $\%$ quaternized DMEAMA (left) and lonic Fluorogel with $30 \mathrm{wt} \%$ quaternized DMEAMA (right). Adsorbent dosage: $10 \mathrm{mg} / \mathrm{L}$. The data points in the figure are an average of 3 experiments and the error bar show their standard deviation.
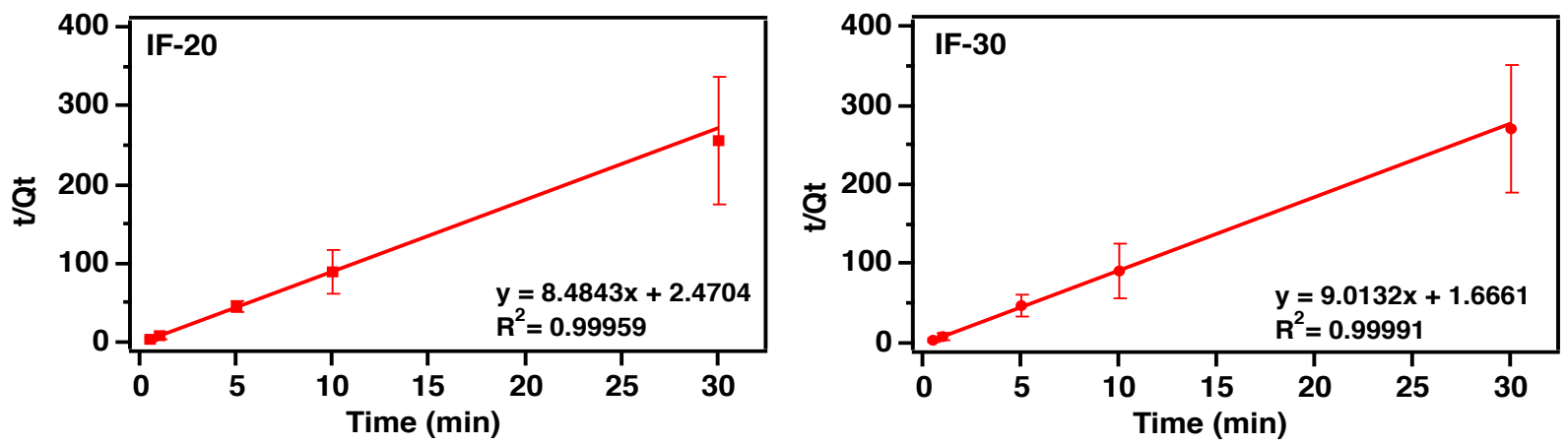

Figure S7: Pseudo second order plots of Ionic Fluorogel with $20 \mathrm{wt} \%$ quaternized DMEAMA (left) and lonic Fluorogel with 30 wt\% quaternized DMEAMA (right). Adsorbent dosage: $10 \mathrm{mg} / \mathrm{L}$; GenX: $1 \mu \mathrm{g} / \mathrm{L}$. The data points in the figure are an average of 3 experiments and the error bar show their standard deviation. 


\subsection{Binding isotherm studies}

The batch isotherm studies were performed in $125 \mathrm{~mL}$ polypropylene bottles (100 mL operating volume) containing a magnetic stir bar on a multi-position stirrer at $23-25{ }^{\circ} \mathrm{C}$ at $500 \mathrm{rpm}$. The deionized water containing the ionic fluorogel adsorbent $(100 \mathrm{mg} / \mathrm{L})$ was stirred for $3 \mathrm{~h}$ before the GenX addition. A stock solution of GenX was spiked to create initial concentrations of $0.2,1,5,10,20,30$ and $50 \mathrm{mg} / \mathrm{L}$. The suspension was stirred for $21 \mathrm{~h}$ to reach equilibrium and an aliquot was taken in a centrifuge tube. The aliquots were centrifuged for 15 minutes and the supernatant from the top was taken for LCMS analysis. High concentration samples were serially diluted (5-10 mg/L diluted 20x and 20-50 mg diluted 100x) before LCMS analysis. Control experiments in the absence of adsorbent were performed under identical conditions to account for handling losses. All the batch experiments were carried out in triplicate.

Langmuir adsorption and Freundlich isotherm fits were generated by Non-linear Least Square Regression of the following equation.

Langmuir adsorption isotherm:

$$
\begin{array}{ll}
q_{e}\left(\mathrm{mg} \mathrm{g}^{-1}\right)=\text { Amount of PFAS adsorbed on the solid phase at equilibrium } \\
q_{e}=\frac{q_{m} K_{L} C_{e}}{1+C_{e} q_{m} K_{L}} \quad \begin{array}{l}
q_{m}\left(\mathrm{mg}^{-1} \mathrm{~g}\right)=\text { Maximum adsorption capacity of adsorbent at equilibrium } \\
C_{e}\left(\mathrm{mg}^{-1} \mathrm{~L}^{-1}\right)=\text { Residual PFAS concentration at equilibrium } \\
K_{L}\left(\mathrm{mg}^{-1} \mathrm{~L}^{-1}\right)=\text { Equilibrium constant }
\end{array}
\end{array}
$$

Freundlich adsorption isotherm:

$$
\begin{array}{ll} 
& q_{e}\left(\mathrm{mg} \mathrm{g}^{-1}\right)=\text { Amount of PFAS adsorbed on the solid phase at equilibrium } \\
& C_{e}\left(\mathrm{mg}^{-1} \mathrm{~L}^{-1}\right)=\text { Residual PFAS concentration at equilibrium } \\
& K_{F}\left(\mathrm{mg} \mathrm{g}^{-1}\right)\left(\mathrm{L} \mathrm{mg}^{-1}\right)^{1 / n}=\text { Freundlich constant. } \\
& n \text { is the intensity of adsorption }
\end{array}
$$

Note: A preliminary fit was generated using linearized equations of Langmuir ( $1 / q_{e}$ vs $\left.1 / C_{e}\right)$ and Freundlich (In $\mathrm{q}_{\mathrm{e}} \mathrm{vs} \ln \mathrm{C}_{\mathrm{e}}$ ) adsorption isotherm and the obtained values were used as a starting point for non-linear least square regression analysis. We used a Microsoft excel spreadsheet template obtained from USAARS website. ${ }^{3}$ 
Table S1: Langmuir and Freundlich parameters derived from linearized plots of the GenX binding isotherm

\begin{tabular}{|c|c|c|c|c|c|c|}
\hline \multirow{2}{*}{ Fluorogel } & \multicolumn{3}{|c|}{ Langmuir Fit } & \multicolumn{3}{c|}{ Freundlich Fit } \\
\cline { 2 - 7 } & $\mathrm{KL}_{\mathrm{L}}\left(\mathrm{M}^{-1}\right)$ & $\mathrm{Qm}(\mathrm{mg} / \mathrm{g})$ & $\mathrm{R}^{2}$ & $\mathrm{~K}_{\mathrm{F}}(\mathrm{mg} / \mathrm{g})(\mathrm{L} / \mathrm{mg})^{1 / n}$ & $\mathrm{n}$ & $\mathrm{R}^{2}$ \\
\hline IF-20 & $5.9 \times 10^{6}$ & 278 & 0.99 & 141 & 2.2 & 0.93 \\
\hline IF-30 & $1.5 \times 10^{7}$ & 217 & 0.99 & 152 & 2.2 & 0.95 \\
\hline
\end{tabular}
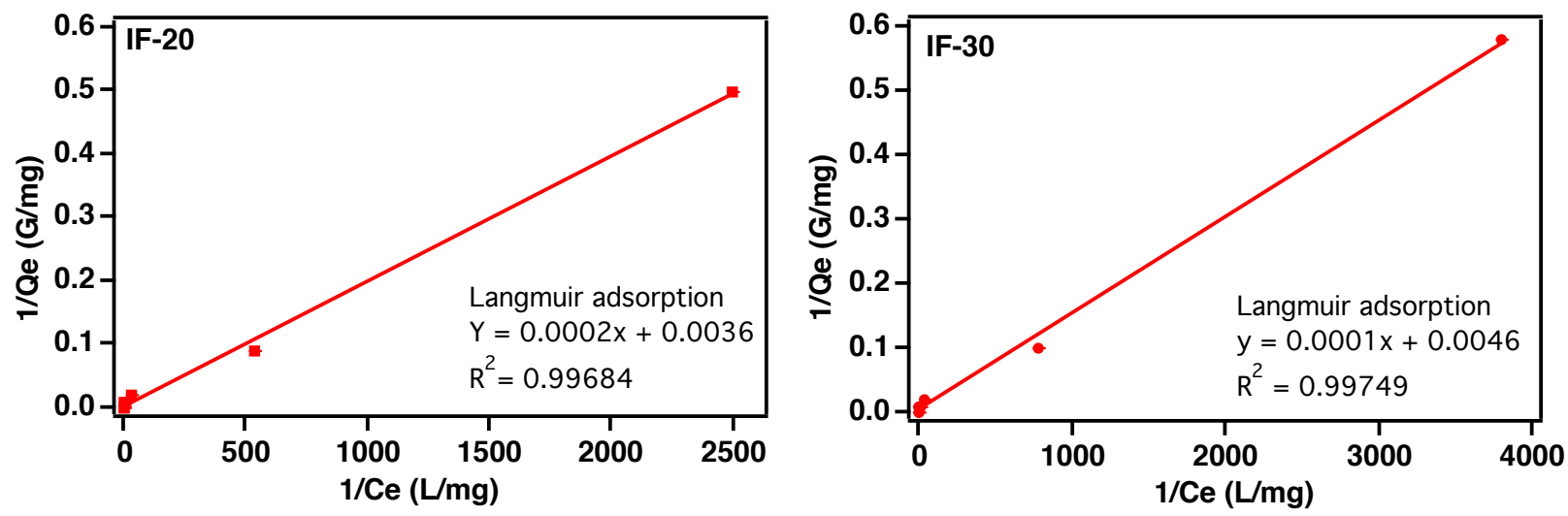

Figure S8: GenX adsorption isotherm linear fitted to Langmuir model for lonic Fluorogel with 20 wt\% quaternized DMEAMA (left) and lonic Fluorogel with $30 \mathrm{wt} \%$ quaternized DMEAMA (right). Adsorbent dosage: $100 \mathrm{mg} / \mathrm{L}$; [GenX]: 0.2-50 mg/L. The data points in the figure are an average of 3 experiments and the error bar show their standard deviation.
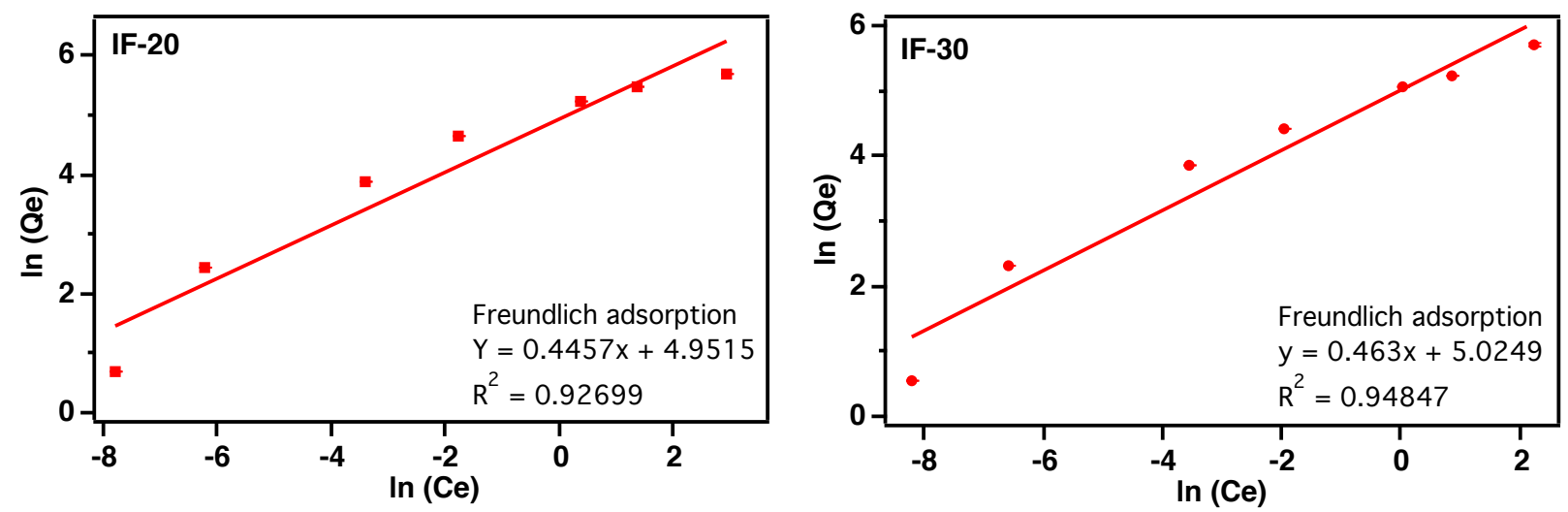

Figure S9: GenX adsorption isotherm linear fitted to Freundlich model for lonic Fluorogel with 20 wt $\%$ quaternized DMEAMA (left) and Ionic Fluorogel with $30 \mathrm{wt} \%$ quaternized DMEAMA (right). Adsorbent dosage: $100 \mathrm{mg} / \mathrm{L}$; [GenX]: 0.2-50 mg/L. The data points in the figure are an average of 3 experiments and the error bar show their standard deviation. 

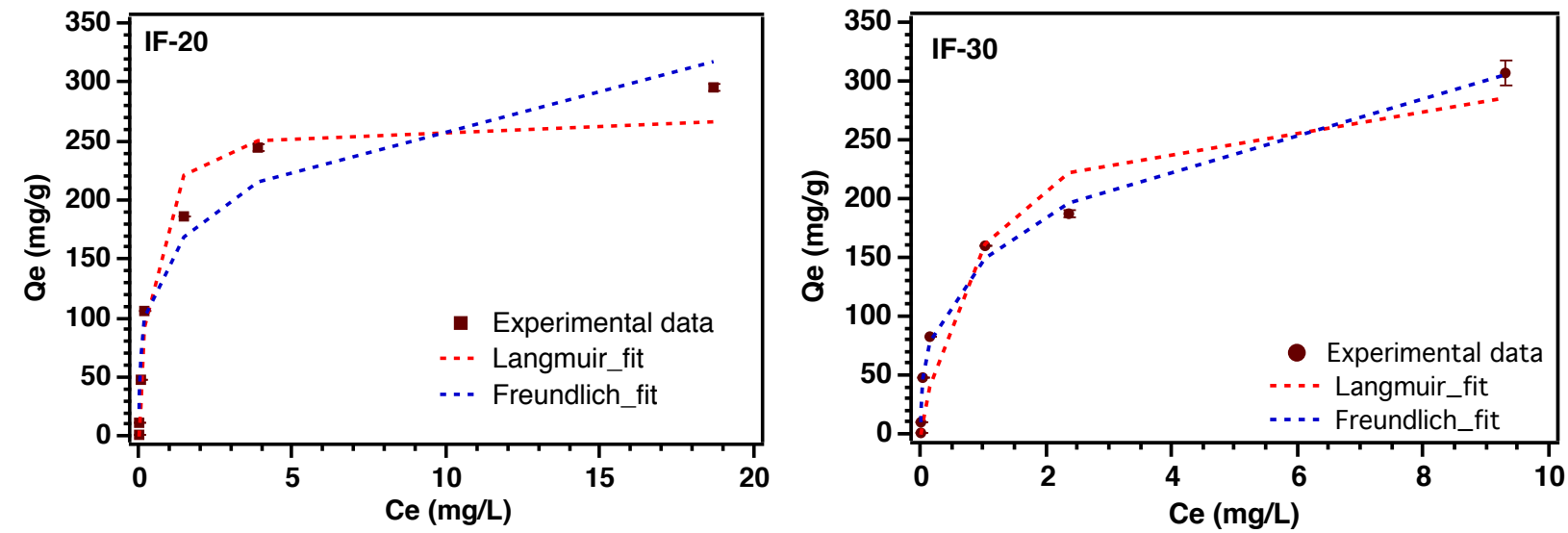

Figure S10: GenX adsorption isotherm for lonic Fluorogel with $20 \mathrm{wt} \%$ quaternized DMEAMA (left) and Ionic Fluorogel with $30 \mathrm{wt} \%$ quaternized DMEAMA (right). Dotted lines represent fit to Langmuir (red) and Freundlich (blue) models. Adsorbent dosage: $100 \mathrm{mg} / \mathrm{L}$; [GenX]: 0.2-50 mg/L. The data points in the figure are an average of 3 experiments and the error bar show their standard deviation. 


\subsection{Real water studies}

The adsorption kinetic experiments were performed in $500 \mathrm{~mL}$ polypropylene bottles equipped with a magnetic stir bar. The experiments were performed at room temperature on magnetic stirrers. The adsorbent dose was set at $100 \mathrm{mg} / \mathrm{L}$ with a total operating volume of $400 \mathrm{~mL}$. The fluorogel was soaked in $5 \mathrm{ml}$ of water for 3 days with occasional sonication to disperse the adsorbent before being adding to the 1 ug/L PFAS spiked water. About $10 \mathrm{~mL}$ aliquot was taken at each predetermined time intervals $(0,30,60$ and 120 mins). The aliquots were filtered through pre-washed 0.45 um glass fiber syringe filter and the filtered solution was analyzed by LCMS to determine the residual PFAS concentration. Two control experiments to account for PFAS losses and PFAS contaminations during handling were performed under an identical condition in the absence of adsorbent and Deionized water. This batch kinetics experiment was performed in duplicate. Settled water was obtained from the Sweeney Water Treatment Plant (TOC = 1.3 $\mathrm{mg} / \mathrm{L}, \mathrm{pH}=6.2$ ).
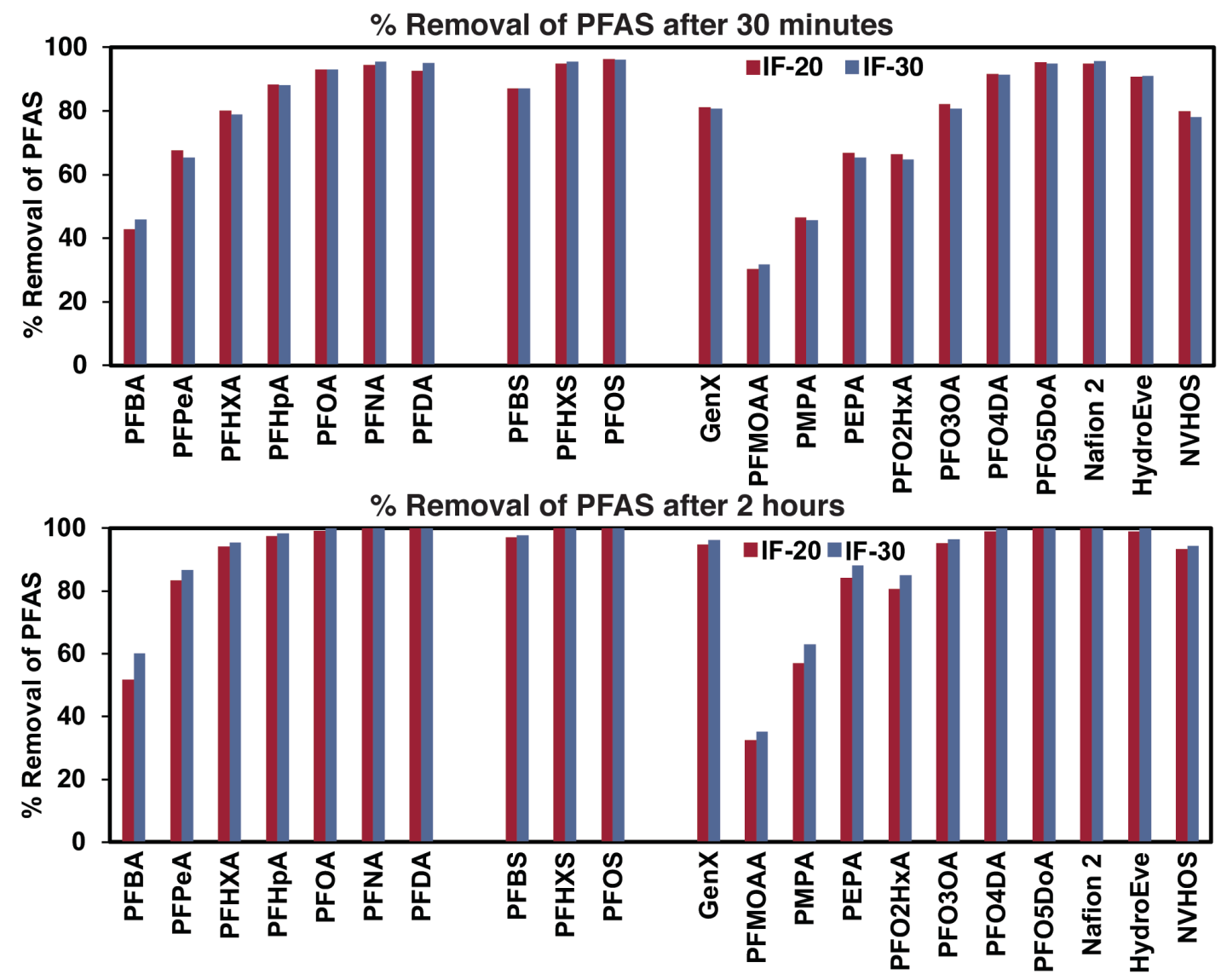

Figure S11: 21 PFAS absorption by lonic Fluorogel with 20 wt \% quaternized DMEAMA (red) and lonic Fluorogel with $30 \mathrm{wt} \%$ quaternized DMEAMA (blue). Adsorbent dosage: $100 \mathrm{mg} / \mathrm{L}$; [PFAS]: $1 \mu \mathrm{g} / \mathrm{L}$ each. The data shown are an average of duplicate experiments. 


\section{ADSORPTION AND REGENERATION STUDIES}

Low Concentration (10 $\mathrm{mg} / \mathrm{g})$ : Adsorption experiment: lonic Fluorogel IF-20+ $(20 \mathrm{mg})$ was suspended in deionized water $(5 \mathrm{~mL})$ followed by series of sonication and vortexing for 5 mins to disperse the adsorbent. The resulting suspension was passed through $20 \mathrm{~mL}$ syringe fitted with $0.45 \mu \mathrm{m}$ PTFE filter ( $25 \mathrm{~mm}$ ), additional water was used if necessary. The IF-20+ thus created a packed bed of resin on top of the PTFE filter through which subsequent water had to flow through in order to exit the syringe. A solution of GenX (10 $\mathrm{mg} \mathrm{L}^{-1}, 20 \mathrm{~mL}$ ) was passed through the filter over 2 mins and the resultant filtrate was collected in a polypropylene tube. The PTFE filter was washed by passing through deionized water $(20 \mathrm{~mL})$ to remove any trace of GenX solution. The change in GenX concentration in the filtrate was measured by LC-MS.

Desorption experiment: The PTFE filter containing GenX was extracted by passing through $20 \mathrm{~mL}$ of a methanolic solution containing $400 \mathrm{mM}$ ammonium acetate over 2 minutes. The concentration of extracted GenX was analyzed by LC-MS. The PTFE filter was washed by passing through deionized water ( $20 \mathrm{~mL})$ to remove any trace of methanolic solution left over and the residual deionized water was removed by vacuum suction.

The adsorption-desorption cycle was extended to 5 cycles to demonstrate the recyclability of the ionic fluorogel without the loss of efficiency.

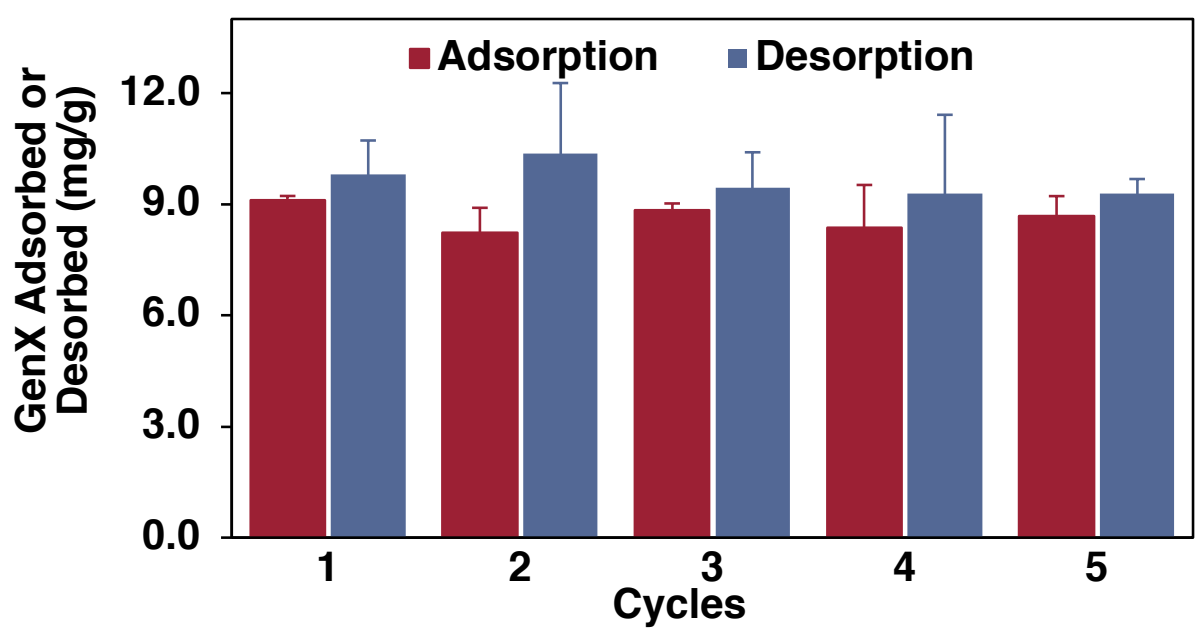

Figure S11: Regeneration and reuse of ionic fluorogel IF-20 with $400 \mathrm{mM}$ methanolic ammonium acetate. Adsorbent dosage: $20 \mathrm{mg}$; [GenX]: $10 \mathrm{mg} / \mathrm{L}, 20 \mathrm{~mL}$. Extraction: IF-20 was extracted with $400 \mathrm{mM}$ methanolic ammonium acetate over 2 minutes.

High Concentration (200mg/g): Adsorption experiment: Ionic Fluorogel IF-20+ $(5 \mathrm{mg})$ was suspended in deionized water $(4.67 \mathrm{~mL})$ in a $20 \mathrm{~mL}$ glass vial followed by stirring 18 hours to disperse the adsorbent. The solution was spiked with $33 \mu \mathrm{L} 30 \mathrm{mg} / \mathrm{mL}$ GenX (final concentration $200 \mathrm{mg} / \mathrm{L}$ ), and the solution was stirred 2 hours. The mixture was transferred to a $15 \mathrm{~mL}$ centrifuge tube and centrifuged at $3000 \mathrm{rpm}$ for 3 minutes. 
An aliquot of the supernatant was used to determine the change in GenX concentration in the filtrate via LC-MS.

Desorption experiment: The pellet was transferred from the centrifuge tube to the glass vial, and $5 \mathrm{~mL}$ methanolic ammonium acetate $(400 \mathrm{mM})$ was added. The mixture was stirred 4 hours, after which the centrifugation process was repeated. The concentration of extracted GenX was analyzed by LC-MS.

The adsorption-desorption cycle was extended to 2 cycles to demonstrate the recyclability of the ionic fluorogel without the loss of efficiency.

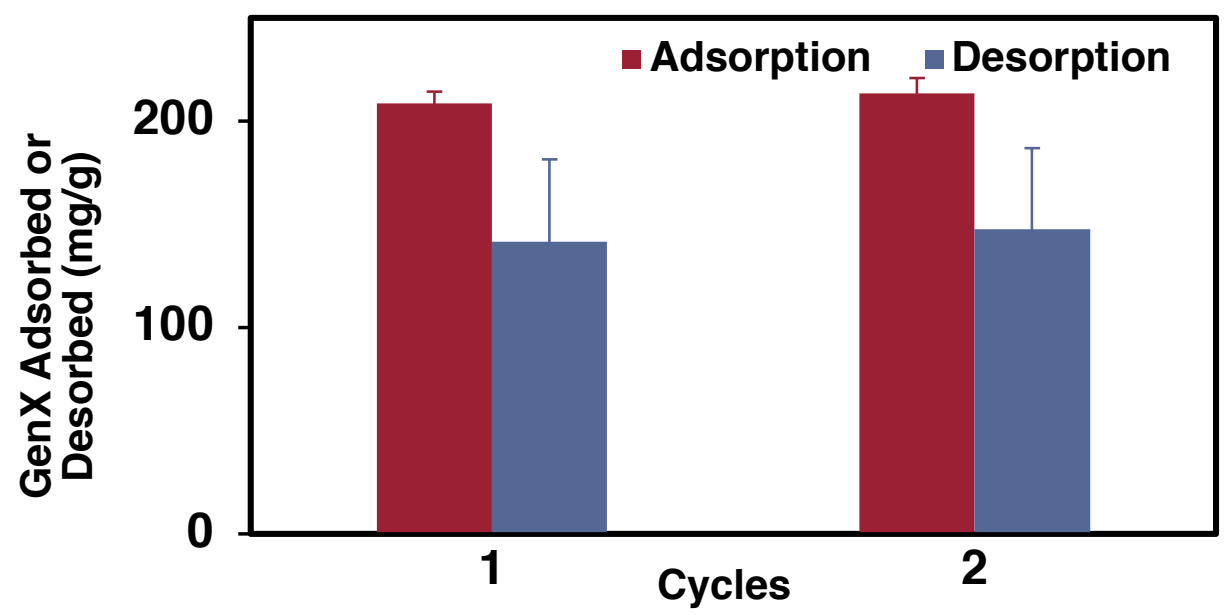

Figure S12: Regeneration and reuse of ionic fluorogel IF-20 with $400 \mathrm{mM}$ methanolic ammonium acetate. Adsorbent dosage: $40 \mathrm{mg}$; [GenX]: $200 \mathrm{mg} / \mathrm{L}, 5 \mathrm{~mL}$. Extraction: IF-20 was extracted with $400 \mathrm{mM}$ methanolic ammonium acetate $(5 \mathrm{~mL})$. 


\section{SYNTHESIS OF FLUOROGEL}

An illustrative procedure for the synthesis of ionic fluorogel IF-20 and IF-20+ is given below:

To a $20 \mathrm{~mL}$ scintillation vial with green top cap equipped with magnetic stir bar was added Fluorolink MD700 (1.6 g, $80 \mathrm{wt} \%$ ), 2-(dimethylamino)ethyl methacrylate (0.4 g, $20 \mathrm{wt} \%$ ), azobisisobutyronitrile (20 mg, $1 \mathrm{wt} \%)$ and trifluoroethanol $(2.0 \mathrm{~g})$. The vial was closed, nitrogen was passed through the solution for 5 minutes, and the solution was heated at $70{ }^{\circ} \mathrm{C}$ for $5 \mathrm{~h}$ while stirring at 200-300 rpm. Within $15 \mathrm{mins}$, gel particles were observed and within $1 \mathrm{~h}$ the entire mixture gelled. After the reaction, the mixture was cooled to room temperature and the gel was ground to fine powder. To this powder, additional trifluoroethanol (10 $\mathrm{mL}$ ) and iodomethane $(2 \mathrm{~mL})$ was added and the mixture was stirred at room temperature for $24 \mathrm{~h}$. The content of the vial was transferred to teabag using ethanol as a transfer solvent. The gel was washed with ethanol using a Soxhlet extraction set up for $24 \mathrm{~h}$. Finally, the gel was dried in a vacuum oven at $50{ }^{\circ} \mathrm{C}$ for $24 \mathrm{~h}$. The dried gel was passed through $125 \mu \mathrm{m}$ and $75 \mu \mathrm{m}$ sieves to collect particles in the size range of $75-125 \mu \mathrm{m}$. The ionic fluorogel IF-20+ was obtained as a pale-yellow powder in $2.2 \mathrm{~g}$ yield.

To obtain fluorogel F-20 (tertiary amine derived), the methylation step was not performed. Instead, after grinding, the fluorogel was directly place in teabag and purified using Soxhlet apparatus.

Other formulations of fluorogel (F) or ionic fluorogel (IF) containing varying amount of amine/ammonium derivatives were prepared by adding appropriate amount of amine and Fluorolink MD700 using the procedure above. For instance, to make IF-30+, $1.4 \mathrm{~g}$ of Fluorolink MD 700 and $0.6 \mathrm{~g}$ of 2(dimethylamino)ethyl methacrylate $(0.4 \mathrm{~g}, 20 \mathrm{wt} \%$ ) was used (yield: $2.3 \mathrm{~g}$ ).

Synthesis of control PEG gel: PEG gels were obtained using the same procedure as mentioned above. The Fluorolink MD 700 was replaced by poly(ethylene glycol) dimethacrylate (average Mn 750). This particular molecular weight was chosen to mimic the number of atoms in the backbone between the dimethacrylate functionality of fluorolink. 


\section{CHARACTERIZATION}

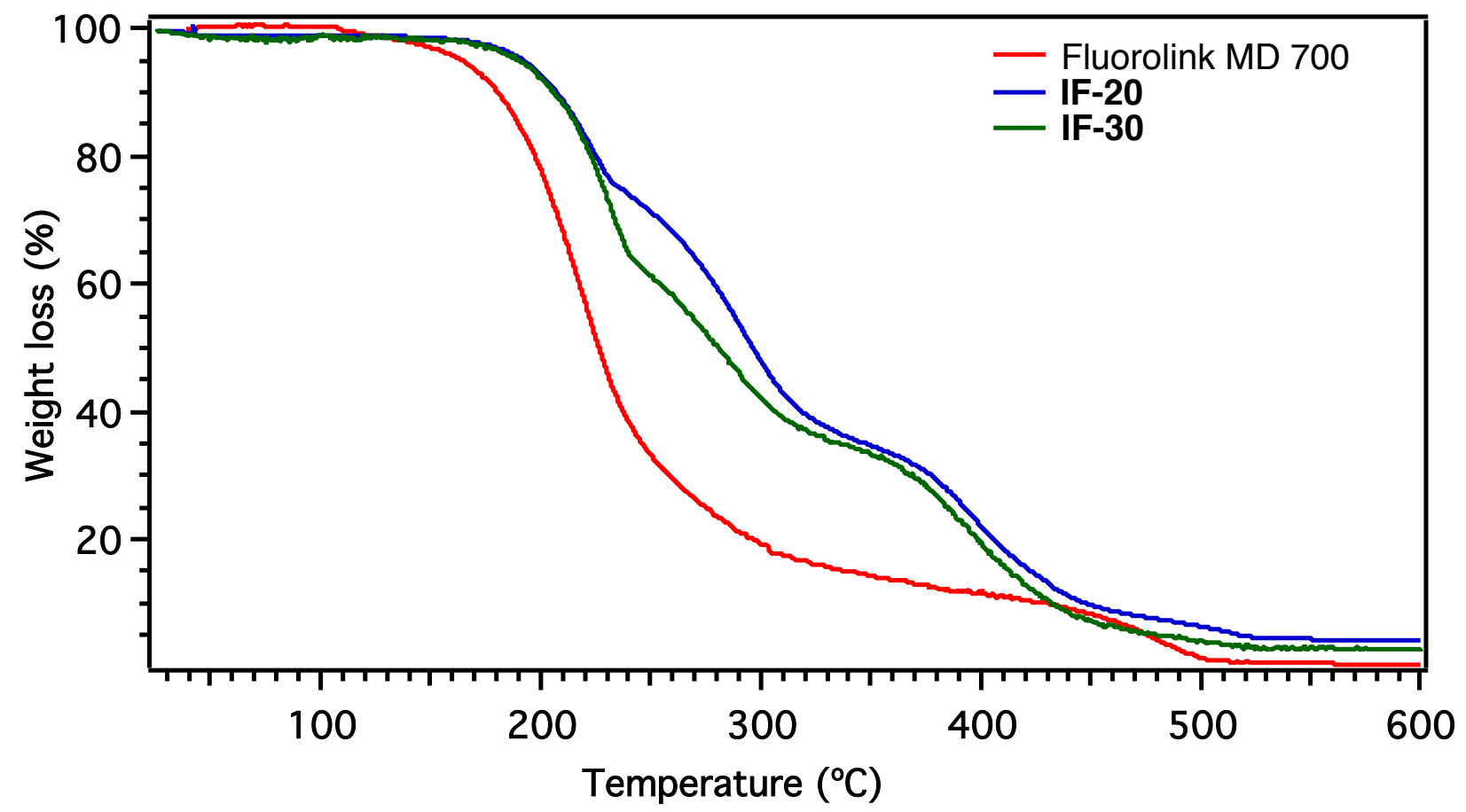

Figure S13: Thermogravimetric analysis (TGA) of Fluorolink MD700 (red), ionic fluorogel IF-20 (blue) and IF-30 (green).

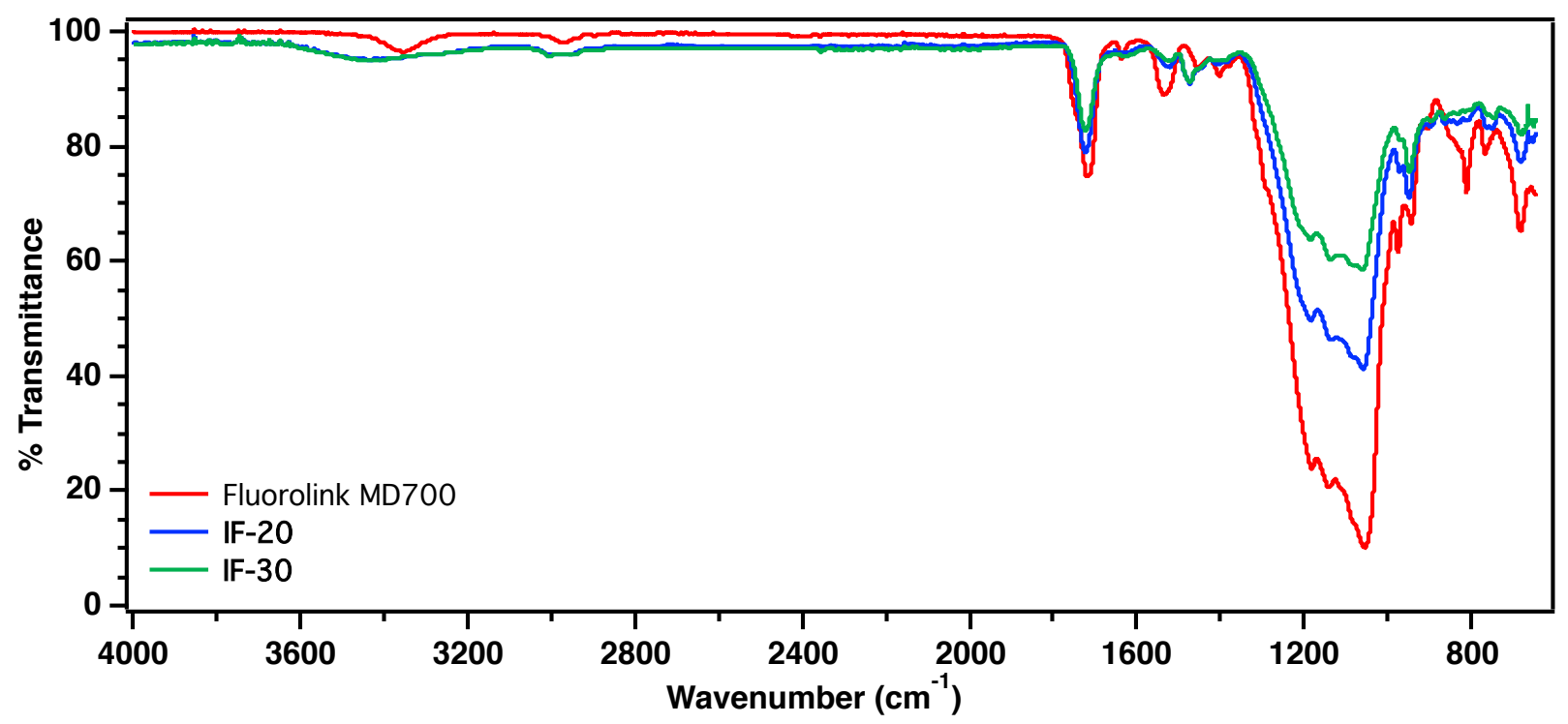

Figure S14: Fourier transform-infrared (FTIR) spectra of Fluorolink MD700 (red) and ionic fluorogel IF-20 (blue) and IF-30 (green). 


\section{REFERENCES}

(1) Sun, M.; Arevalo, E.; Strynar, M.; Lindstrom, A.; Richardson, M.; Kearns, B.; Pickett, A.; Smith, C.; Knappe, D. R. U. Environ. Sci. Technol. Lett. 2016, 3, 415-419.

(2) Ho, Y. S.; McKay, G. Process Biochem. 1999, 34, 451-465.

(3) https://www.ars.usda.gov/midwest-area/bowling-green-ky/food-animal-environmentalsystems-research/people/carl-bolster/sorption-isotherm-spreadsheet/. 